Portland State University

PDXScholar

1972

\title{
Kinetics of the Cope rearrangement of 3,4-diphenylhexa-1,5-diene
}

Harlan Albert Jerome Berg

Portland State University

Follow this and additional works at: https://pdxscholar.library.pdx.edu/open_access_etds

Part of the Analytical Chemistry Commons, Inorganic Chemistry Commons, and the Materials Chemistry Commons

Let us know how access to this document benefits you.

\section{Recommended Citation}

Berg, Harlan Albert Jerome, "Kinetics of the Cope rearrangement of 3,4-diphenylhexa-1,5-diene" (1972). Dissertations and Theses. Paper 1116.

https://doi.org/10.15760/etd.1116

This Thesis is brought to you for free and open access. It has been accepted for inclusion in Dissertations and Theses by an authorized administrator of PDXScholar. Please contact us if we can make this document more accessible: pdxscholar@pdx.edu. 
AN ABSTRACT OF THE THESIS OF Harlan A. J. Berg for the Master of Science in Chomistry presented September 1, 1972.

Title: Kinetics of the Cope Rearrangement of 3,4-Diphenylhexa-1,5-diene

APPROVED BY MEMBERS OF THE THESIS COMMTTEE

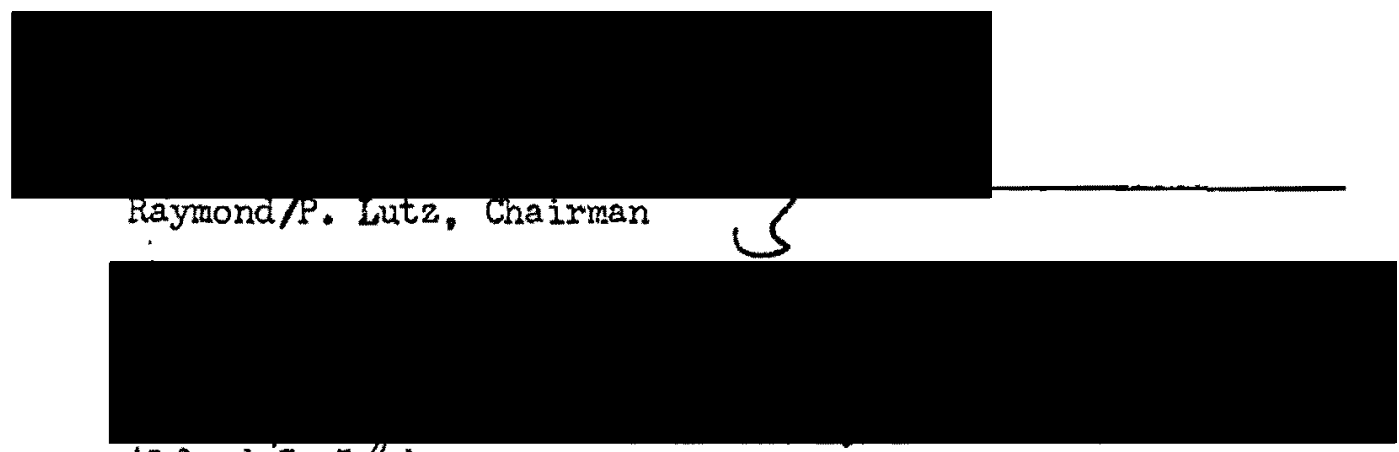

Alfred S. Lffinson

Kinetics investigations of the thermal Cope rearrangement of meso- and dl-3,4-diphenylhexa-1,5-dienes were undertaken in order to gain information about the transition states for these reactions by determining the appropriate enthalpies and entropies of activation. of particular interest were the activation parameters for the meso. compounds' rearrangement as it represents the only known example in which both four- and six-centered transition states are of comparable energy .

Kinetics of the $d l$ isomers' rearrangement in the terperature range $90-110^{\circ}$ were determined using $5 \times 10^{-5} \mathrm{M}$ solutions of the olefin in heptane contained within sealed Pyrex arapules. Extents of reaction were determined from UV absorbance measurements at $256 \mathrm{~nm}$. Froduct 
instability, becoming pronounced after 85-90\% reaction, was observed. Two rate constants were calculated covering $0.85 \%$ and $0.98 \%$ reaction. respectively, from each set of data. Evaluations of enthalpies of activation from the two sets of rate constants gave, respectively, 23.8 and $23.9 \mathrm{kcal} / \mathrm{mole}$, indicating that inclusion of the increasingly erratic kinetics data collected after $85 \%$ reaction had little effect upon this activation parameter. The corresponding entropies of activation at $100^{\circ}$ were -12.9 and -12.6 eu. Both activation parameters are consistent with those for other Cope rearrangements. indicating that the thermal isomerization of dl-3,4mdiphenylhexa-1,5diene in the temperature range $90-110^{\circ}$ is normal in every respect. Kinetics studies of the more interesting meso-diene vere precluded by the failure to locate means of controlling product decomposition, which would be expected to become more severe at the higher temperature required $\left(140-160^{\circ}\right)$.

Additionally, alunina-catalyzed isomerizations first observed accidentally for the dl-diene, and subsequently verified for both meso- and dl-olefins, were examined. In contact with 100 times its weight of very active alumina at room temperature the dl isomer was found to undergo conversion in the extent of $40 \%$ during a two-hour period to trans, trans-1,6-diphenylhexa-1,5-diene. meso-3,4-Diphenylhexa-1,5-diene underwent structural reorganization more slowly; aftor 70 hours over alumina a $15 \%$ conversion to a $9: 1$ mixture of cisitransand trans, trans-1,6-diphenylhexa-1,5-dienes was noted. The stereospecificities observed for these unusual catalyzed rearrangements parallel those of their thermally. induced counterparts. 
Modifications in the syntheses of meso- and dl-1,6.dibromo-3,4diphenylhexanes, and meso- and dl-3,4-diphenylhexa-1,5-dienes, are also presented. 
KINETICS OF THE COPE REARRANGEMENT OF 3,4-DIPHENKLHEXA-1,5-DIENE

by

HARLAN ALBERT JEROME BERG

A thesis submitted in partial furfillment of the requirements for the degree of

MASTER OF SCIENCE

in

CHEMISTRY

Portland State Untversity

1972 
TO THE OFFICE OF GRADUATE STUDIES AND RESEARCH:

The members of the Committee approve the thesis of

Harlan A. J. Berg prosented September 1, 1972.

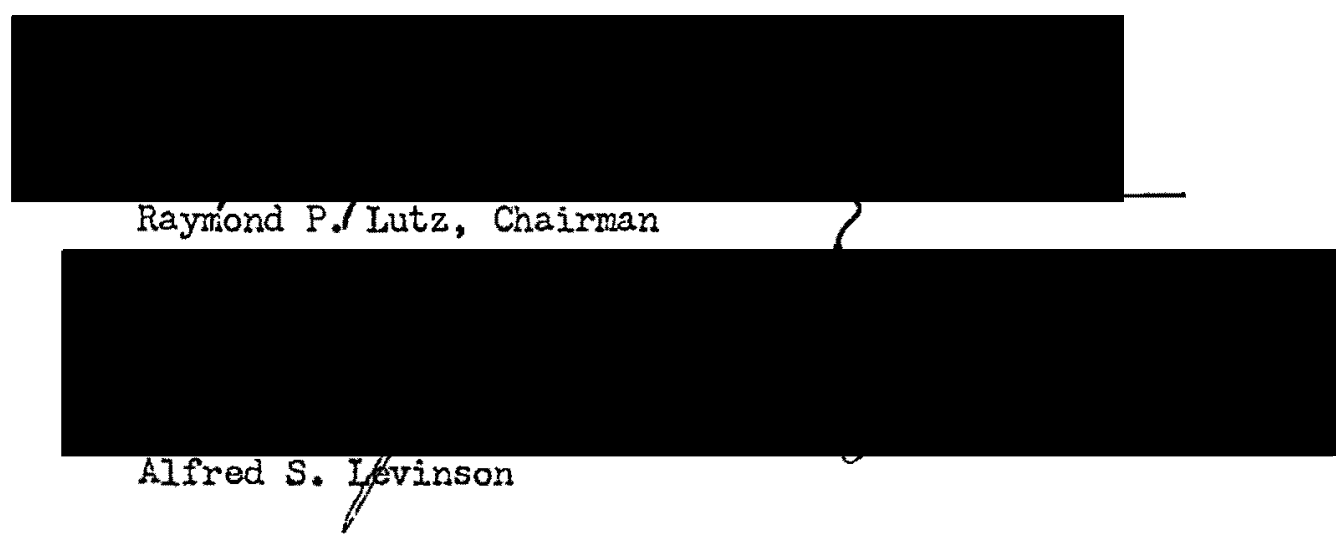

APPROVED:
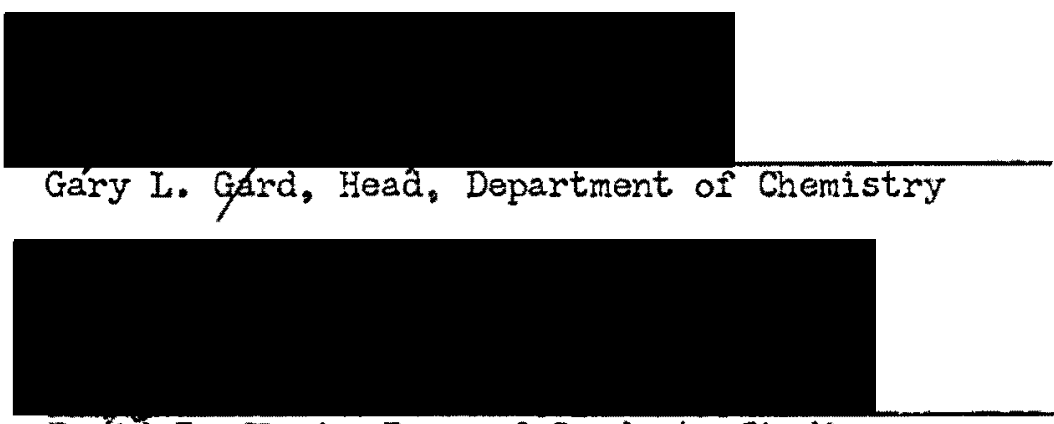

David T. Clark, Dean of Graduate Studies 


\section{ACKNOWLEDGMENTS}

The author is indebted to, his research director Dr. Raymond P.

Lutz for his patient (and persistent) guidance during the course of the investigations described herein, and to Ms V.R. Berg who sacrificed much time and effort in the preparation of this manuscript. In addition thanks are extended to Mr. Thomas Brandt and Ms. A. Cook who manufactured ampules and other glass apparatus required for the experimental work. 
TABLE OF CONTENTS

PAGE

ACKNOWLELGMENTS. . . . . . . . . . . . . . . .

LIST OF TABLES ...................... . . . v

LIST OF FIGURSS. . ................... vi vi

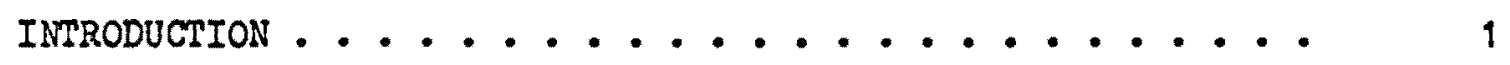

DISCUSSION ........................

I PREPARATION OF STARTING MATERTALS . . . . . . $10^{\circ}$

II KINETICS OF THE THERMAL COPE REARRANGEMENT OF . . . DI-3,4-DIPHENYLHEXA-1,5-DIENE . . . . . . 12

III ALUMINA-CATALYZED REARRANGEMENT . . . . . . . . 22

EXPERTMENTAL . . . . . . . . . . . . . . 25

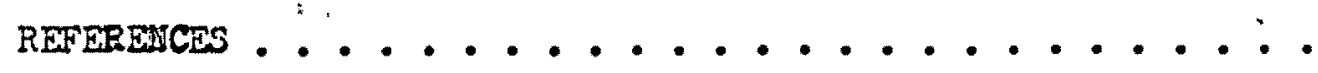

APPEADICES

A RAW KINETICS DATA . . . . . . . . . . .

B EVAIJATIONS OF KINETICS DATA AND ACTIVATION 
IIST OF TABLES

MABLE

PAGE

I Sumary of Rate Constants for Rearrangement of dl-3,4Dlphenylhexa-1,5-diene ................ 18

II Surmary of Activation Parameters for Rearrangement of dI-Diene and Related Reactions .......... 


\section{LIST OF FIGURES}

FIGURE

PACE

1 The Structures of Barbital. Phenobarbital, and a Representative Vinylic Analogue ..........

2 Preparation of Ethyl (1-Mothyl propenyl)-allylcyanoacotate. . . . . . . . . . . . .

3 A Simplified Mechanism for the Cope Rearrangement. . 4

4 Alternative Transition States for the Cope Rearrangement IIlustrated by the Isomerization of a meso3,4-Disubstituted Hexa-1,5-diene ........

5 Rearrangements of meso- and al-3,4-Dimethylhexa-1,5-

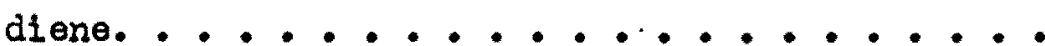

6 Synthetic Sequence Inployed in Preparing meso- and dl3,4-Diphenylhexa-1,5-diene as IIlustrated for the Meso Isomer. ....................

7 Representative Plots of Absorbance versus Time IIlustrating Decomposition of trans,trans-1,6-Diphenylhexa-1,5mdiene Solutions Heated in Different Environments ...................

8. Representative First-Order Kinetics plots for the Thermal Cope Rearrangement of dl-3,4-Diphenylhexa-1,5diene at $90^{\circ}, 100^{\circ}$, and $110^{\circ}$.........

! A Arrhenius Flot of $-\operatorname{Ln}(k / T)$ versus $1 / T$ Employing Rate - Constants: Caloulated from Data Collected up to $98 \%$ Reaction ................. 
1.0 Arrhenius Plot of $-\operatorname{In}(k / t)$ vorsus $1 / T$ Employlng Rate Constants Calculated from Data Collected up to

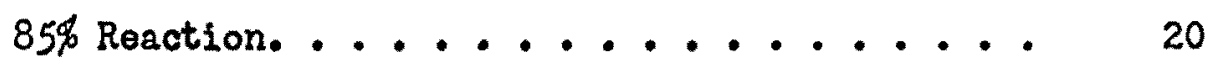

11 Cuvette Wash-Bottlo ............... 26

12 Apparatus for Draining Ampules. . . . . . . . 39

13 Apparatus for Evacuating Ampules. . . . . . . 39

14 IMR Spectrun of Crude Product from Attempted Preparation of meso-3,4-Diphenylhexa-1,5-diene During Which Base-Catalyzed Rempt getpent Occured. . . 52

15 Gas Chromatogram of meso Rearranged Neat at $220^{\circ}$ for $15 \mathrm{~min} . . . . . . .53$

16 Gas Chromatogram of the 4:1 v/v Heptane-Benzene Eluate Residue from Rearrangement of meso-3,4-Diphenylhexa-1.5-diene on Alumina ...........

17 Gas Chromatogram of Heated $\left(220^{\circ}, 15 \mathrm{~min}\right) 4: 1 \mathrm{v} / \mathrm{v}$ Heptane-Benzene Elluate Residue from Rearrangement of meso-3,4-Diphenylhexa-1,5-diene on Alumina.. 


\section{IMTRODUCTION}

During the first four decades of this century considerable research effort was channeled into the development of satisfactory general methods for the synthesis of vinylic analogues of barbital and phenobarbital, shown in $51 g .1$, since the former had been found to possess marked hypnotic powers. Investigative attention was focused<smiles>CCC1(CC)C(=O)NC(=O)NC1=O</smiles>

a

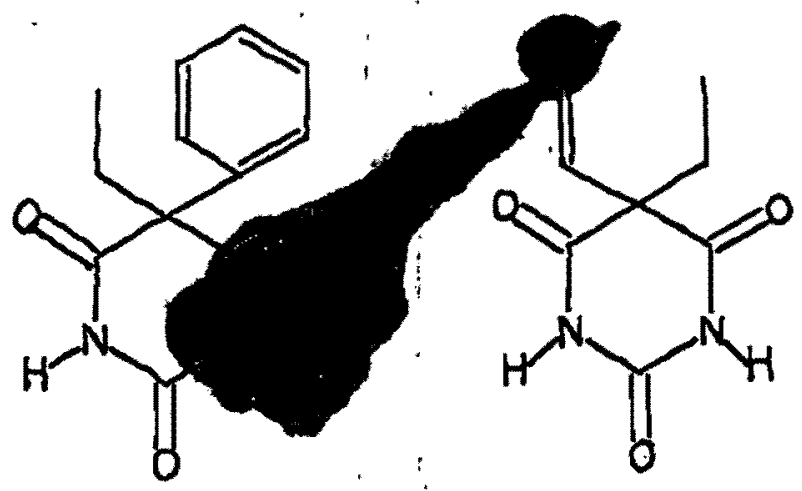

b c

Figure 1. The structures of barbital (a), phenobarbital (b), and a representative vinglic analogue $(c)$.

upon appropriately substituted malonic and cyanoacetic esters, and malononitriles, as these are the immediate precursors of barbituric acid and many of its derivatives. Numerous synthetic routes to these compounds were proposed and surveyed. Most, however, proved impractical due either to extensive formation of undesired side products or to the absence of any reaction whatever (1). The remainder yielded the sought after vinyl-substituted compounds in only slightly more than iqolable quantities (2).

In the latter pert of the 1930's however, the late Arthur C. Cope 
and co-workers came upon several highly sucessful methods for the preparation of unsaturated malonic and cyanoacetic esters $(3,4)$ which involved the alkylation of delocalized carbanions generated from alkylidene malonates or cyanoacetates by treatment with strong bases. One súch compound, ethyl (1-methylpropenyl)-allylcyanoacétate, II, prepared from allyl bromide and the sodio derivative of ethyl (1-methylpropylidene)-cyanoacetate, I, (see Fig.2), was found to behave rather peculiarly when purified by repeated fractional distillation in vacuo. Each successive fractionation was accompanted by an increase

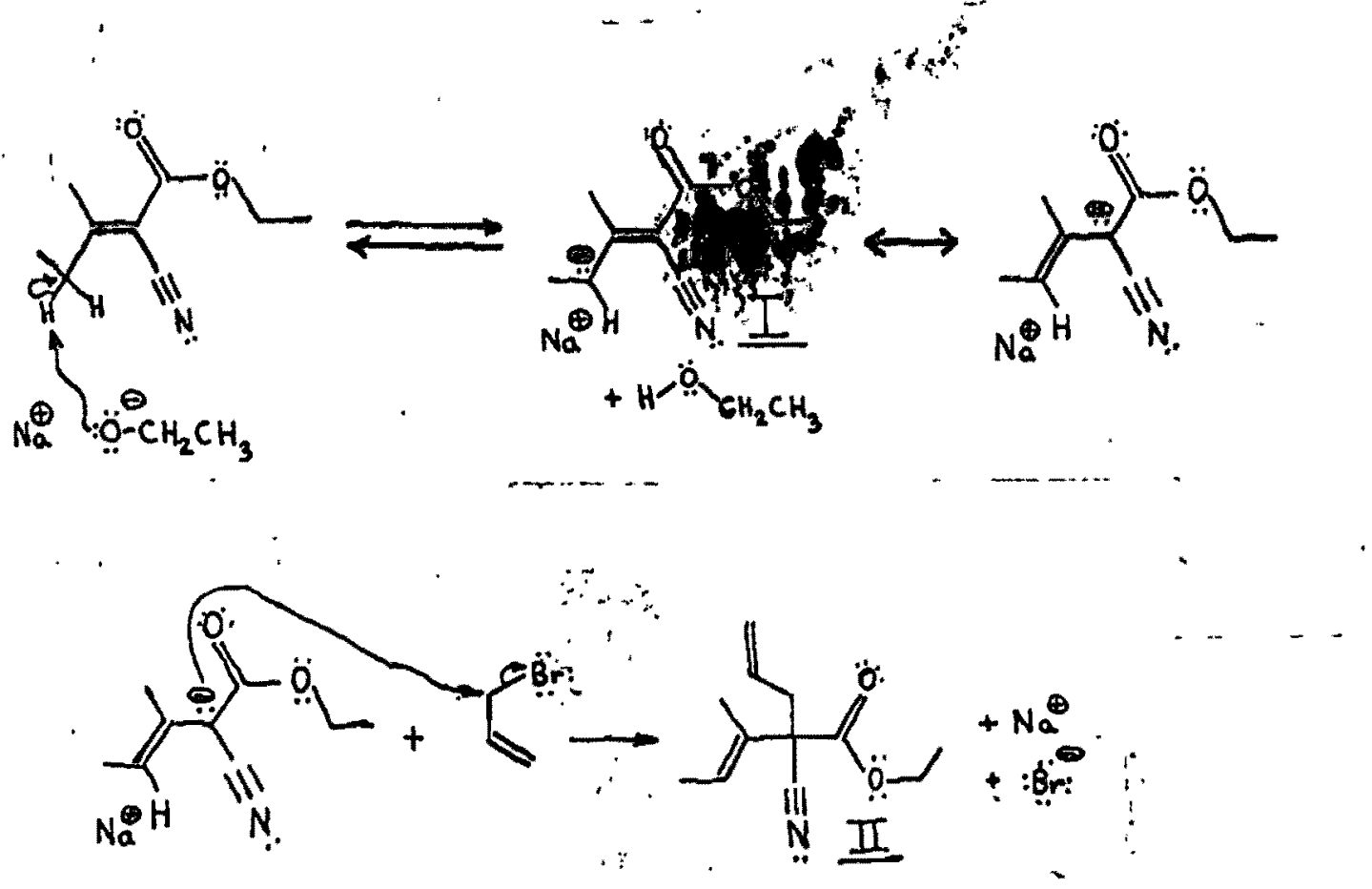

Figure 2. Preparation of ethyl (1-methylpropenyl)-allylcyanoacetate.

in bolling point and index of refraction, both eventually becoming constant. Based upon these observations and others (4), Cope concluded that the initially formed (1-methylpropenyl)-allylcyanoacetate which could be insolated in good yleld when distillation was carried 
out at low pressures, had undergone a skeletal reorganization. Additional evidence allowed Cope to formulate the rearrangement product as ethyl (1,2-dimethyl-4-pentenylideno)-cyanoacetate, III (no steriochemistry implied).

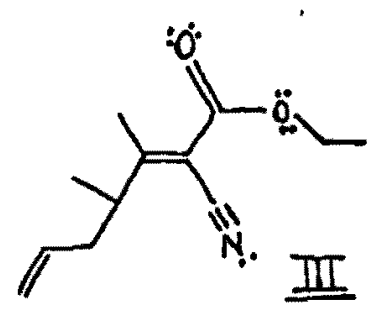

By analogy to the Claisen rearrangenent, in which an allyl group migrates from an electronegative atom (axygen) to a more electropositive atom (carbon), Cope proposed that the allyl group migrated from one carbon of the cyanoacetate, riade electronegative by the adjoining cyano and carbethoxy functionalities, to another more electropositive carbon three atomic centers distant. In addition the reaction was found to depend upon the presence of an allylic group (4). as in the Gaisen rearrangement, leading Cope to postulate a cyclical mochanism in which the allylic group migrated intramolecularly and with inversion (see Fig. 3). (An energy profile for the proposed mechanism is also shown for completeness.)

Subsequent investigations by Cope and co-workers revealed that this thermally induced reaction was general for allylic-vinylic cyanoacetates, malononitriles, and malonic esters (5), as well as stmucturally similar substrates activated by only one cyano or carboxyl group (6), or by a single phenyl group (7), and substrates which were unactivated (7). In every Instance migration of the allylic group 


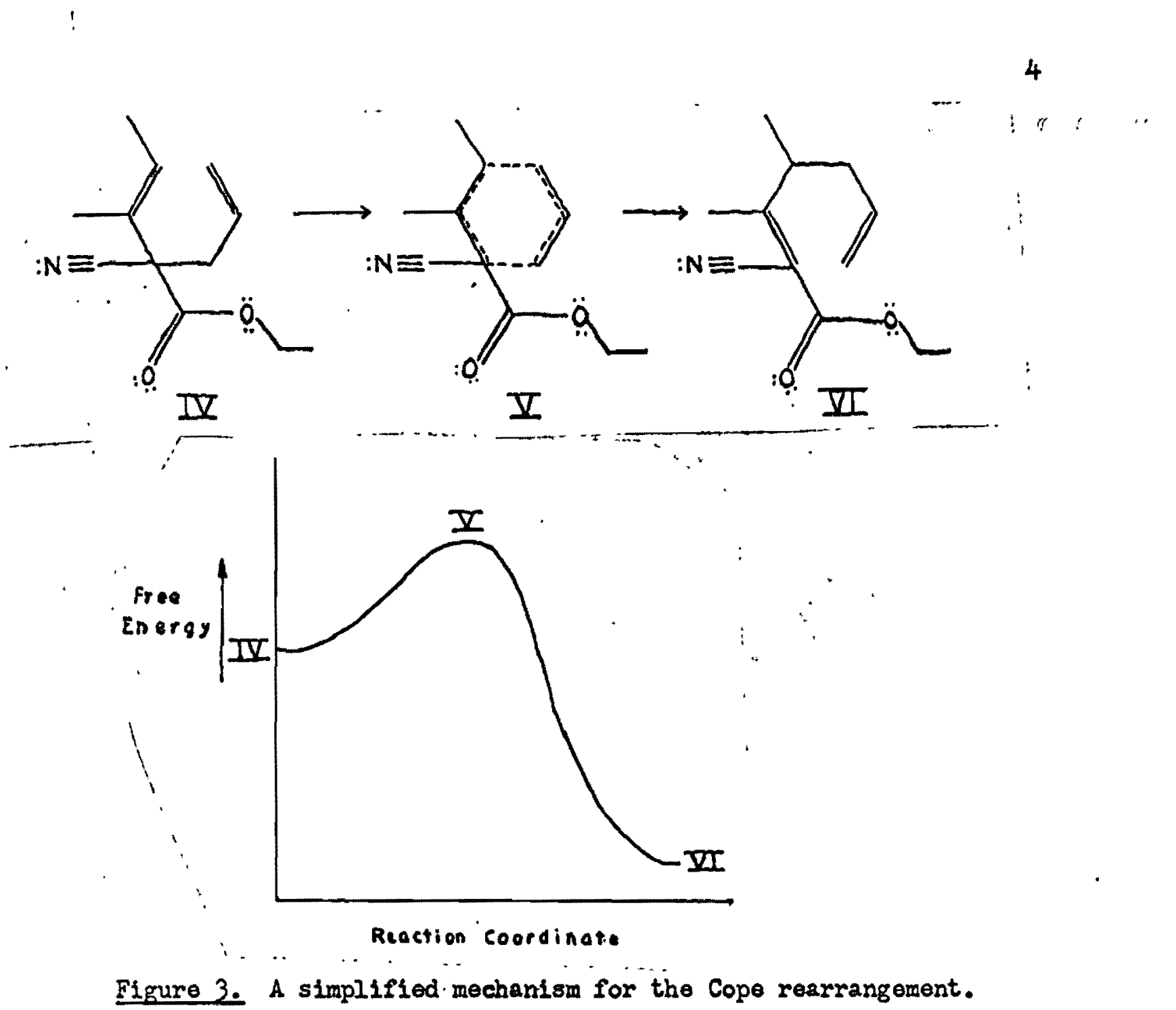

occured from the original binding site to a new atomic position two atoms distant. That the shift was accompanied by inversion of the 2llylic molety and preceded by an intramolecular process was demonstrated by product analysis and crossing experiments (8).

Subsequent to Cope's discovery, the mechanism of the thermally induced valence isomertzation of compounds possessing a hexa-1,5-dienetype carbon skeleton that is, the Cope rearrangement has been subjected to much scmutiny. The bulk of information acquired thus far overwhelmingly favors a concerted mechanism (9). The evidence includes 
the absence of intermolecular crossing $(8,9)$, insensitivity to solvent changes $(10,11)$, the adherence of reaction rates to the first-order rate law, and the inversion of allylic groups. Consistant also with the constrained, low-polarity transition state which such a mechanism demands are the relatively low enthalpies of activation, and negative volumes and entropies of activation, which are observed experimentally. Values of the former vary between about $6 \mathrm{kcal} / \mathrm{mole}$ and $36 \mathrm{kcal} / \mathrm{mole}$. Typical entropies of activation 110 in the range of -5 to -14 eu, (cal/mole-degree). (A summary of activation parameters with their associated references may be found in the discussion section.)

Examination of an acyclic molecule possessing a hexa-1,5-dienetype structure indicates however that more than one six-membered cyclic transition state is possible; each of these is derived from a different conformation of the hexadiene skeleton (Fig. 4). One of the

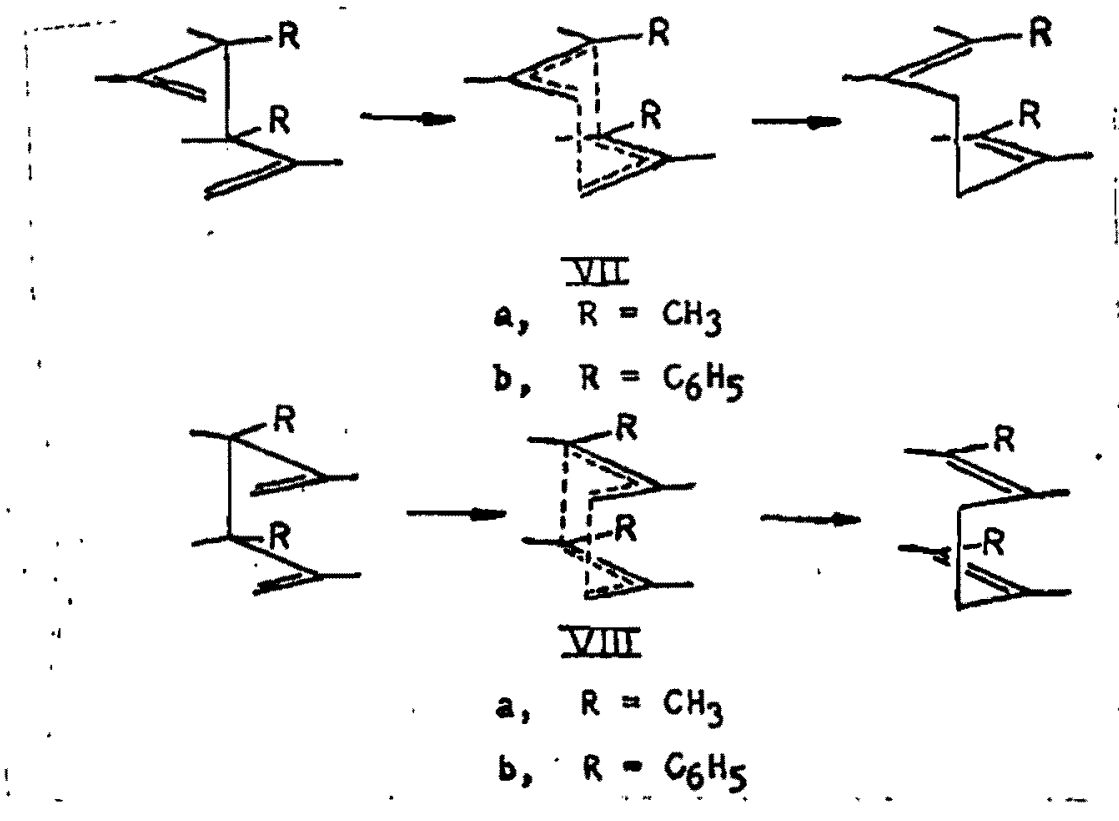

Figure 4. Alternative transition states for the Cope rearrangement illustrated by the isomerization of a meso-3,4-disubstituted hexa-1,5-diene. 
more general questions raised concerning the mechanism of the Cope rearrangerent is which of these transition states is energetically preferred. An answer was provided by Doering and Roth (12) who examined the rearrangement of meso-and di-3,4-dimthylhexa-1,5-diene (Fig. 5). The meso compound, IX, rearranged almost exlusively (99.7\%) to cis, trans-octa-2,6-diene, $x$. The remaining $0.3 \%$ may have been trans, trans 1somer, XII, arising from impurities in the starting material. The isomeric dl-diene, XI, yielded $90 \%$ trans,trans- and $10 \%$ cis,cis2,6-octadienes-XII and XIII respectively.

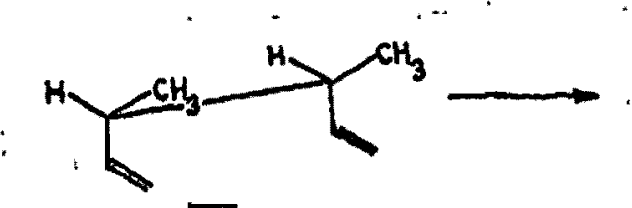

IX
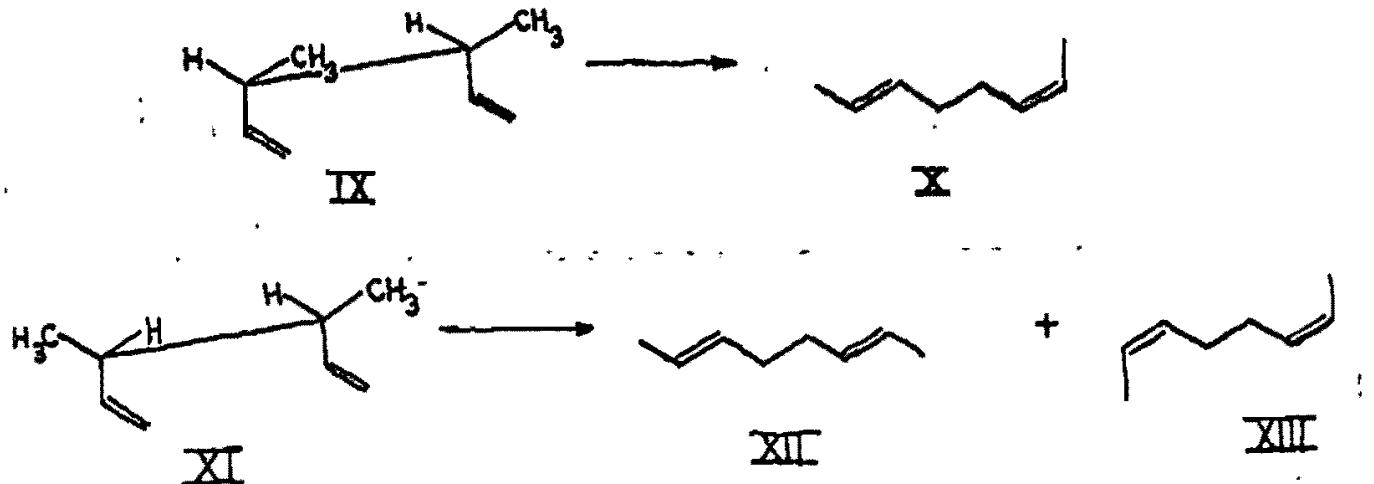

XI

XII

XII

Fjgure 5. Rearrangements of meso- and d]-3,4-dimethylEvxa-1.5-diene.

Doering's results may be rationalized by considering the rearrangement of the meso-dimethyl compound as illustrated above in. Fig. 4. Of the two possible transition states avallable, only the chair-Iike four-centered-type, VIIa, leads to the major product, cis,tans-octa-2,6-diene. The six-centered boat-like transition state, VIIIa, leads to the isomerio trans,trans-diene which, observed in trace amounts, may have resulted from residual dl isomer in the starting material. A similar analysis starting with the dl compound (not shown 
in Fig. 4) indicates that both rearrangement products were generated Via chair-like transition states differing only in the disposition of substituents. The product of reaction through a six-centered transition state, cis,trans-octa-2,6-diene, was not observed.

Based upon the observed product distributions for these highly stereospecific rearrangements Doering concluded that the chair-like four-centered transition state of the type VIIa was favored energetically over the boat-like six-centered alternative VIIIa. The difference in energies amounted to at least $5.7 \mathrm{kcal} / \mathrm{mole}$. At this juncture it should be noted that, although neither of the compounds investigated by Doering and Roth underwent significant rearrangement through a six-centered transition state, numerous examples are known in which, due to molecular constraints, reaction is allowed to proceed only through this mode.

Approximately 15 years prior to Doering and Roth's investigations Koch (13) reported that preparation of meso-3,4-diphenylhexa-1,5-diene (shown in Fig. 4,R phenyl) and its rearrangement to a $3: 2$ mixture of trans, trans-1,6- and trans-1,4-diphenylhexa-1,5-dienes. On the basis of the presently known behavior of the Cope, neither of these two products were those which would have been predicted. Preferential formation of the trans,transudiene over the cis,trans isomer contradicted Doering's results, and formation of the trans-1,4 isomer was inconsistent with the established mechanism of the rearrangement. A reinvestigation of this anomalous reaction by Iutz and co-workers (14) revealed that one of Koch's structural assignments was incorrect. In fact the rearrangement product (at $120^{\circ}$ ) consisted of $63 \%$ cis, trans- 
and a still unexpectedly high $37 \%$ of trans,trans-1,6-diphenylhexa-1,5diene as the kinetically controlled products. Evidently both the four-centered and the six-centered transitions states, shown as VIIb and VIIIb, respectively, in Fig. 4, were used. The fallure of this rearrangement to parallel that observod by Doering was attributed to an unfavorable steric interaction accompanying the formation of a cis double bond in the cis, trans 1somer, which could arise only through the four-centesipd transition state (VIIb in Fig. 4). This interaction prevented coplanarity of contiguous phenyl and allyl groups, thereby prohibiting their energetically favorable electronic interaction during reaction. In contrast, the six-centered transition state (VIIIo in Fig. 4) although lacking the fundamental electronic advantages of the four-centered, permitted coplanarity of the aryl and allyl groups. The energetic balance thus obtained between both transition states is such that rearrangement may take place with neaxly equal facility through either. (The two transition states differ only by about 0.4 kcal/mole at $120^{\circ}$ based upon relative rearrangement rates.) Extention of the investigation to include the rearrangement of dl-3.4-diphenylhexa-1,5-diene revealed that the latter underwent isomerization exclusively to trans, trans-1,6-diphonylhexa-1,5-diene through a fourcentered transition state. The absence of the cis,cis isomer predicted to be a product on the basis of Doering's work was attributed to unfavorable steric effects in the four-centered transition state required for its formation.

As the conclusion to the work, kinetics investigations of the rearrangements of both meso- and dl-3,4-diphenylhexa-1,5-diene were 
undertaken to determine activation parameters for the rearrangements. Owing to the simplicity of the dl's rearrangement, its kinetics were investigated first and are reported in the next section. The principal objective of these kineties studies was to evaluate and compare activation parameters for the meso-diene's dual reaction pathways. However problems of product instability at the concentrations used and attempts to solve them precluded study of the more interesting meso-diene.

During the course of purification by chromatography on alumina of a sample of the dl-diene for subsequent use in kinetics work, it was observed that the dl had undergone rearrangement in a manner paralleling the thermal isomerization. A similar rearrangement of the meso-diene was also discovered. These appear to be the first reported examples of alumina-catalyzed Cope rearrangements.

Discussions of the aluminamcatalyzed isomerizations as well as improvements in the synthesis of starting materials are presented in the next section. 


\section{DISCUSSION}

\section{PREPARATION OF STARTIMG MATERIAIS}

meso- and dl-3,4-Diphenylhexa-1,5-diene were prepared via the general synthetic route employed by Lutz (14), as outlined for the meso isomer below (Fig. 6). Tho method of preparation of the starting
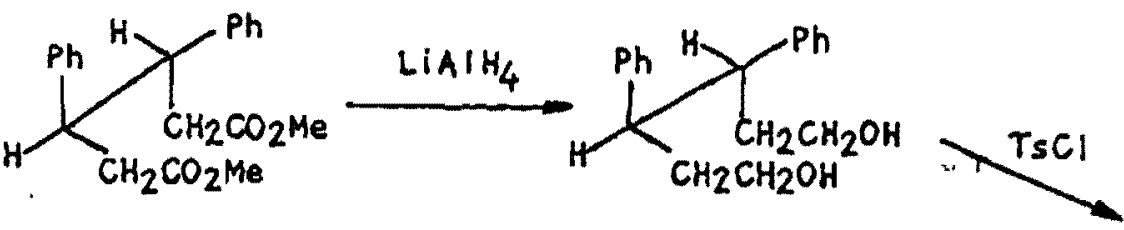

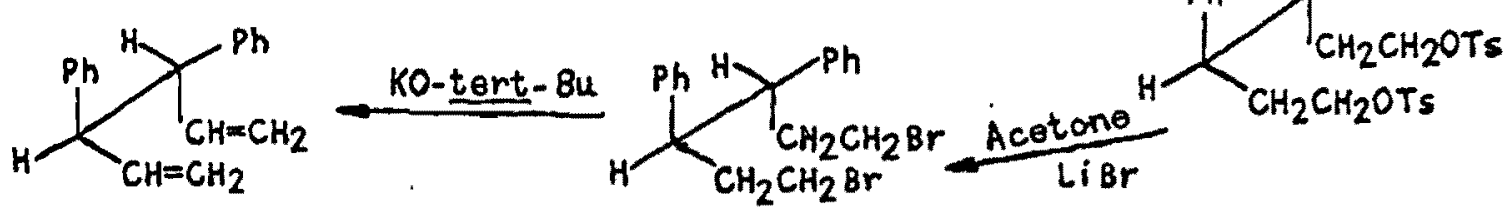

Figure 6. Synthel1e sequence -mployed in prepering meso- and...
dl-3.4-diphenylhexa-1.5diene as illustrated for the meso isomer.

dimethyl- meso- and $\underline{\text { d }}-\beta, \gamma$-diphenyladipates by reductive coupling of mothyl trans-cinnamate over aluminum-amalgam in moist ether proved largely unsatisfactory for several reasons: Its nearly uncontrolable exothermicity, and the modest yields of coupling products obtained (30-40\%) together with the inherent separation problems, were foremost among them. Alternative coupling reactions, effected either electrolytically or with potassium-amalgam (15) offer the advantage of being less exothermic and should be explored if the synthesis is to be repeated. The yields obtainable are not much greater, however, than those from the coupling over aluminum. 
Nucleophilic bromination of the intermediate tosylates also gave low yields due presumably to the presence of excessive amounts of moisture. However, solvent acetone and lithium bromide when used under increasingly more nearly anhydróus conditions resulted in steadily declining conversion. The use (out of pure curiosity) of undried acetone and lithium bromide handled without special precautions to exclude moisture, accelerated the reaction and boosted yields past the approimately $80 \%$ values obtained by Lutz (14) to 95-99\%. Apparently some moisture is necessary to allow the reaction to proceed rapidly to near completion.

Elimination of the meso-bromide with large excesses of potassium tert-butoxide in a $1: 1$ ( $\mathrm{vol}$ ) dimethyl sulfoxide-tert-butyl alcohol solvent gave a substantial proportion of ether as a side product from the competing $S_{N}{ }^{2}$ substitution reaction, together with other products (based upon the poor recovery from dry-column-chromatographic purification of the crude dienes). Similar behavior was noted for the dlbromide. In an attempt to lessen the amounts of ether formed, dimethyl sulfoxtde alone was employed as solvent for the elimination. An increase in the ratio of elimination to substitution was indeed observed. However the ease with which previously unobserved side products formed under the newly devised elimination conditions made this alternative procedure appear less promising than had been originally anticipated. The preparation of the mesomdiene was especially troublesome in this respect. One attempted elimination of the meso-bromide yielded rearrangement products exclusively (seo experimental section). An NMR 
spectrum of the oily crude product is provided in Fig. 14 in the experimental section. No attempt was made to further establish the composition of the rearrangement product. Based upon the observed splitting patterns and chemical shifts the product may be formulated as a 3,4-diphenylhexa-2,4-diene. The proton ratios, however, are not in agreement with those deduced for the 2,4-hexadiene. It was noted also, on the basis of extents of conversion, that the elimination of the meso-bromide proceeded at a rate estimated to be approximately 10 to 40 times that observed for its dI counterpart under comparable conditions. Differing conformational preferences of the starting materials may account for this observation. However owing to experimental problems the exact correspondence of reaction conditions could not be guaranteed; consequently the variation in rates may be largely artifactual. A wethod of effecting elimination at the expense of substitution employing tosylates as substrates and tetram-n-butylammonium oxalate as the eliminating reagent was described recently by Corey and Terashima (16). Due to the mild conditions used and the absence of any substitution products, this preceedure recomends itself highly as an alternative to either of the alkoxide-promoted eliminations described above.

II. KINETICS OF THE THERMAL COPE REARRANGEMENT OF DL-3,4-DIPEENYLHEXA-1,5-DIENE

The kinetics of the thermal rearrangement of dl-3,4-diphenylhexa-1,5-diene were carried out at 90, 100, and 110 . Runs in triplicate were carried out at each temperature. "In a typical run 
14 Pyrex ampules, filled with aliquots of an approximately $5 \times 10^{-5} \mathrm{M}$ solution of dl-diene in n-heptane and sealed under vacuum, were placed in a stirred oil bath whose temperature was constant to $\pm 0.01^{\circ}$. Arpules were periodically withdrawn from the bath, quenched by cooling in a chloroform bath at $20^{\circ}$ by oV at $256 \mathrm{~nm}$. Values of $-\ln \left(c^{c} / \mathrm{c}_{0}\right)$, where $\% c_{0}$ is the fraction of dl-diene remaining after any period of reaction, were celculated from the expression shown below (Appendix B)

$$
-\operatorname{In}\left\{c_{0}-\left[\left(A-674.4 c_{0}\right) / 35160\right]\right\}+\ln c_{0} \text {. }
$$

A plot of $-1 n\left(c / c_{0}\right)$ versus time was constructed and the slope determined as that for a first order reaction by means of an unweighted least-squares analysis. The data covered extents of reaction from 0 to $98 \%$. Beyond $85 \%$ (three half-lives) the graphed data became increasingly erratic. Control experiments employing trans,trans-1,6diphenylhexa-1,5-diene revealed that product instability was responsible for the behavior. Several corrective methods were investigated. in attempts to eliminate or reduce decomposition of the product trans, trans-1,6-diphenylhexa-1,5-diene. Included among these methods were the admixture of free-radical inhibitors (diphenylamine, N-phenyl- $\beta$ - naphthylamine, and di-n-butylamonium picrate), acids (acetic), or bases (di-n-butylamine) with the trans, trans solutions, heating of the ampules in a gas flame while evacuated and preliminary cleansing of the ampules with hot, aqueous hydrochloric acid, ammonia, or sodium hydroxide solutions, or with $\underline{n}$-heptane. None of the measures examined produced both satisfactory control of decomposition (1..e. restricted it to less than $0.5 \%$ ) and reproducable results (Fig. 7). In addition, kinetics runs carried out at $100^{\circ}$ using di solutions which 
were $5 \times 10^{-7} \mathrm{M}$ in $\mathrm{N}$-phenyl- $\beta$-naphthylamine gave rate constants 1-. dentical within experimental exror to those obtained from runs utilizing untreated samples. The use of inhibitor concentrations high enough to significantly retard decomposition (about $10^{-5} \mathrm{M}$ or greater) was found to be incompatable with the analytical method employed due to the greatly increased absorption at $256 \mathrm{~nm}$. This made analysis of mixtures containing trans, trans-diene in amounts greater than about $3 \times 10^{-5} \mathrm{M}$ impossiblo.

In viow of these results the simple expedient of rinsing ampules with heptane, which proved as effective in moderating decomposition as any other technique examined, was utilized in the kinetics runs. It should be emphasized, however, that no satisfactory method for limiting the trans, trans decomposition was found.

There exist nevertheless several promising alternative techniques, some recently reported, which bear investigation. Included are the poisoning of catalytic glass surfaces by heating in contact with oxygen in a gas-oxygen flame (17); preconditioning of the ampules by prolonged contact with this rearrangement products prior to introduction of a kinetics sample and the use of other glasses (e.g., lead-potash) in the fabrication of sample containers (18).

It should be pointed out here that decomposition of the rearrangement product was not a surface catalyzed reaction, as was shown by analysis of kinetics data obtained using ampules whose surface-tovolume ratio had been increased oight-fold. The development of a satisfactory solution to the decomposition problem is a prerequisite to investigation of the meso-diene's rearrangement kinetics, since 
decomposition would be expected to become more severe at the higher temperatures required $\left(140-160^{\circ}\right)$. In view of the observed product decomposition it was felt desirable to calculate a second set of rate constants using only the first ten'data points corresponding to 70-90\% roaction from each run: beyond these limits deviation of the kinetics plots from linearity became pronounced. Graphical representations of typical kinetics data are shown in Fig. 8. A sumary of the rate con stants obrained is shown in Table I. Crude kinetics data are collected in Appendix A.

Activation enthalpies were calculated from the slopes of plots of $-\ln (k / T)$ versus $1 / T$ where $k$ is the specific reaction rate constant at the absolute temperature T (19). Both sets of rate constants were erployed (Figs. 9,10). Slopes were evaluated through the least squares program utilized for determining the rate constants: enthalpies of activation wero taken as the product of these values and the gas constant. Entropies of activation were calculated at 90,100 , and $110^{\circ}$ from the expression $\Delta S^{\ddagger}=\Delta H^{\ddagger} / T+R I n(k h / A T)$ where $\Delta S^{\ddagger}$ and $\Delta H^{\ddagger}$ are the extropy and enthalpy of activation respectively, $T$ is the absolute temperature, $R$ is the gas constant, $k$ is the rate constant at temperature $T$ and $h$ and $h$ are the constants of Planck and Boltzmann, respectively (19). The enthalpies of activation are found to be 23.9 and $23.8 \mathrm{kcal} / \mathrm{mole}$, as calculated from rate constants whose values had been determined using all experimental data points, and only the first 10 points, respective1y. The corresponding activation entropy values at $100^{\circ}$ are -12.6 and -12.9 eu, respectively. These values are summarized in Table II along with several others representing different Cope rearrangements. It may 


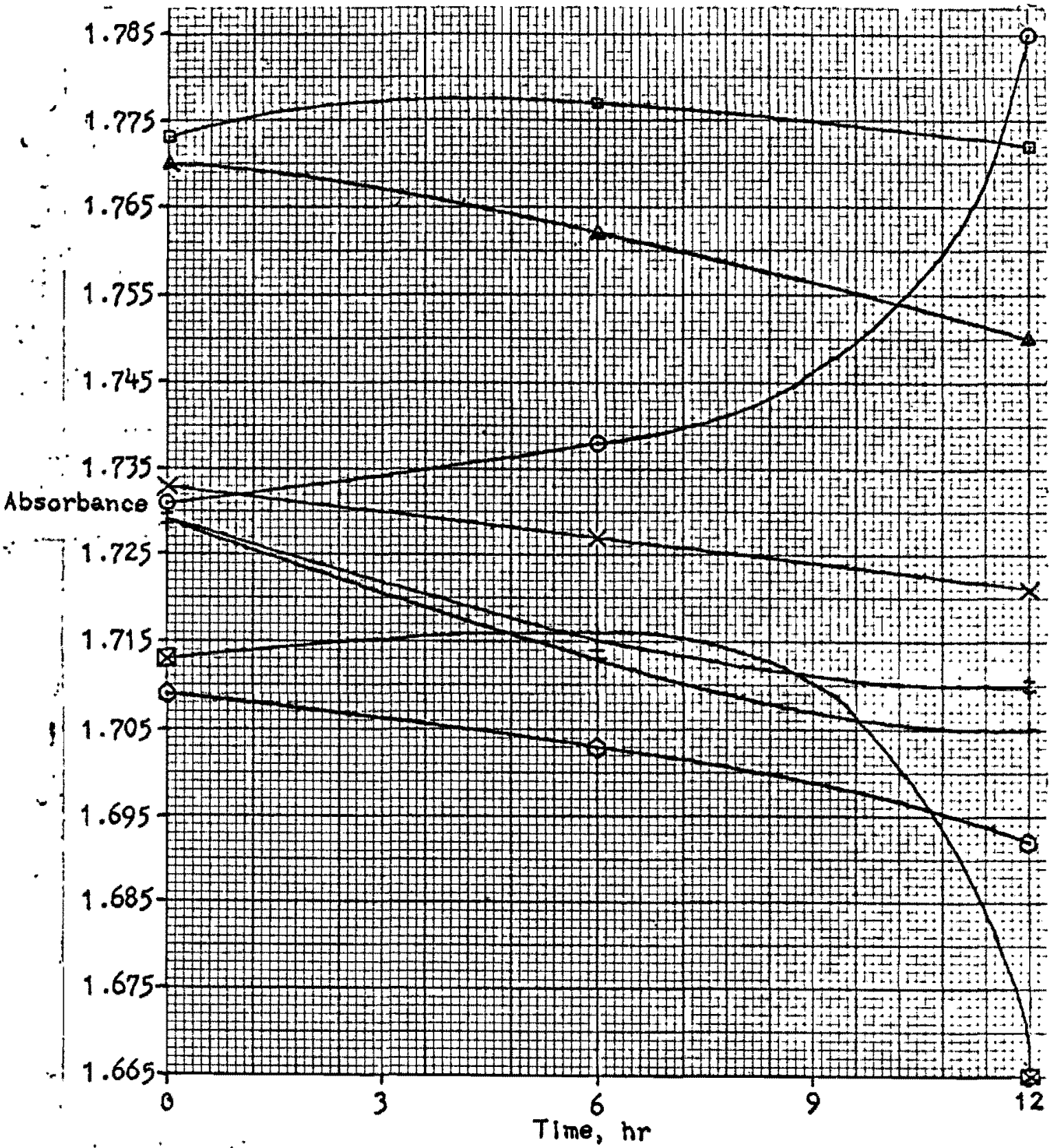

Figure ? Representative plots of absorbance versus time f.Justrating decomposition of trans, trans-1,6-diphenylhexa-1,5-diene solutions heated in different environments. Data are takeil. from run 4 at $90^{\circ}$ as described in the experimental section.

- Ampules rinsed with cone $\mathrm{HCl}$ then $\mathrm{H}_{2} \mathrm{O}$.

- Ampules rinsed with $6 \mathrm{M}$ aqueous $\mathrm{NaOH}$.

$\triangle$ Ampules heated while evacuated until flaremoff observed.

$X$ Ampules rinsed with heptane.

$+D i-n$-butylamnonium picrate added ( $1 \% \mathrm{w} / \mathrm{w}$ of trans, trans present).

\# Hydroquinone added $(1 \% \mathrm{w} / \mathrm{w})$.

OAcetic acid added ( $1 \% \mathrm{w} / \mathrm{w})$.

QDi-n-butylamine added $(1 \% \mathrm{w} / \mathrm{w})$. 
be seen that both the entropies and enthalpies of activation for the thermal rearrangement of da-3,4-diphenylhexa-1,5-diene lie within the expected ranges: this rearrangement is, therefore, in all respects a normal Cope. The stabilizing effects of the aromatic groups upon the transition state is apparent from the moderate enthalpy of activation observed, which is only approximately twomthirds that for the reaxrangement of hexa-1,5-diene itself (1.e.., 1,1-dideuteriohexa-1,5diene).

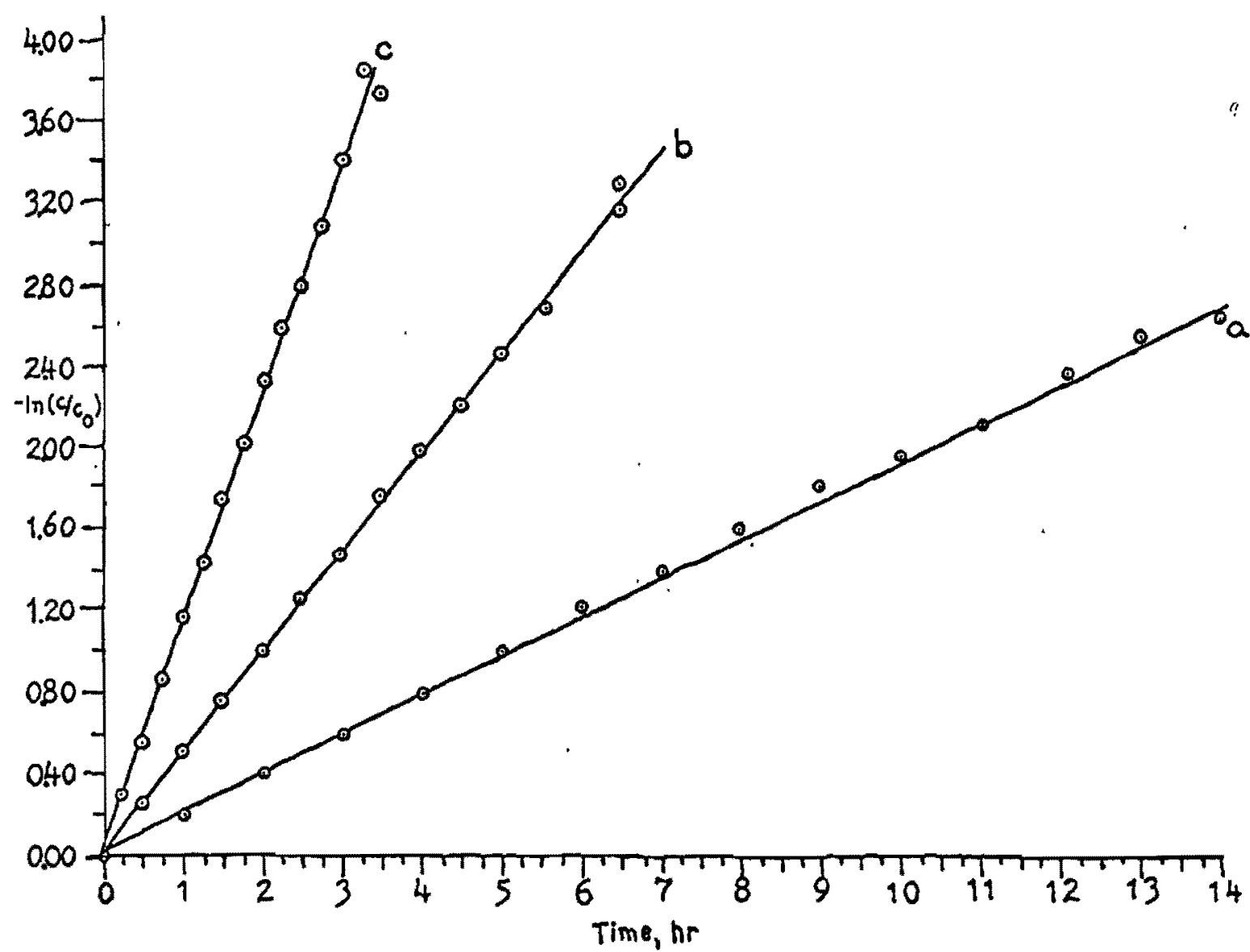

Figure 8. Representative first-order kinetics plots for the thormal Cope rearrangement of dl-3,4-diphenylhexa-1,5-diene at $90^{\circ}(\mathrm{a}), 100^{\circ}$ (b), and $110^{\circ}$ (c). 
TABLE I

SUMMARY OF RATE CONSTANTS

FOR REARRANGEMENT OF

dl-3,4-DIPHENI-

HEXA-1,5-DIENE

Riun number

Rate constant ${ }^{2} \times 10^{4}, \sec ^{-1}$

Temp, ${ }^{\circ} \mathrm{C}$

1

0.544

$(0.554)$

90.02

2

0.531

$(0.546)$

90.03

3

0.534

$(0.551)$

89.90

1

1.31

(1.36)

100.01

2

$1.32^{b}$

(1.38)

100.02

3

1.36

(1.36)

100.02

4

$1.37^{\mathrm{c}}$

(1.36)

100.02

5

1.36

(1.38)

100.02

3.26

(3.31)

109.90

1

3.10

$(3.21)$

109.90

2

$(3.15)^{d}$

109.89

${ }^{2}$ Rate constants calculated using only the first ten experimental points: (averaging $85 \%$ conversion) are shown within parentheses. The remainder were calculated from data collected up to $98 \%$ conversion. amine.

bamples contained approximately $5 \times 10^{-8} \mathrm{MN}$-phenyl- $\beta$-naphthyl-

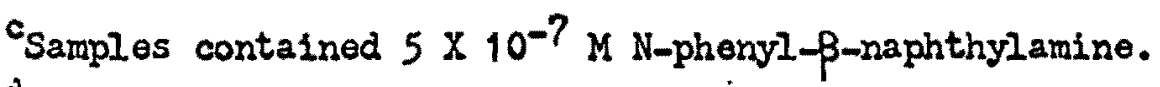

$d_{\text {Surface-to-volume ratio increased 8-fold. }}$ 


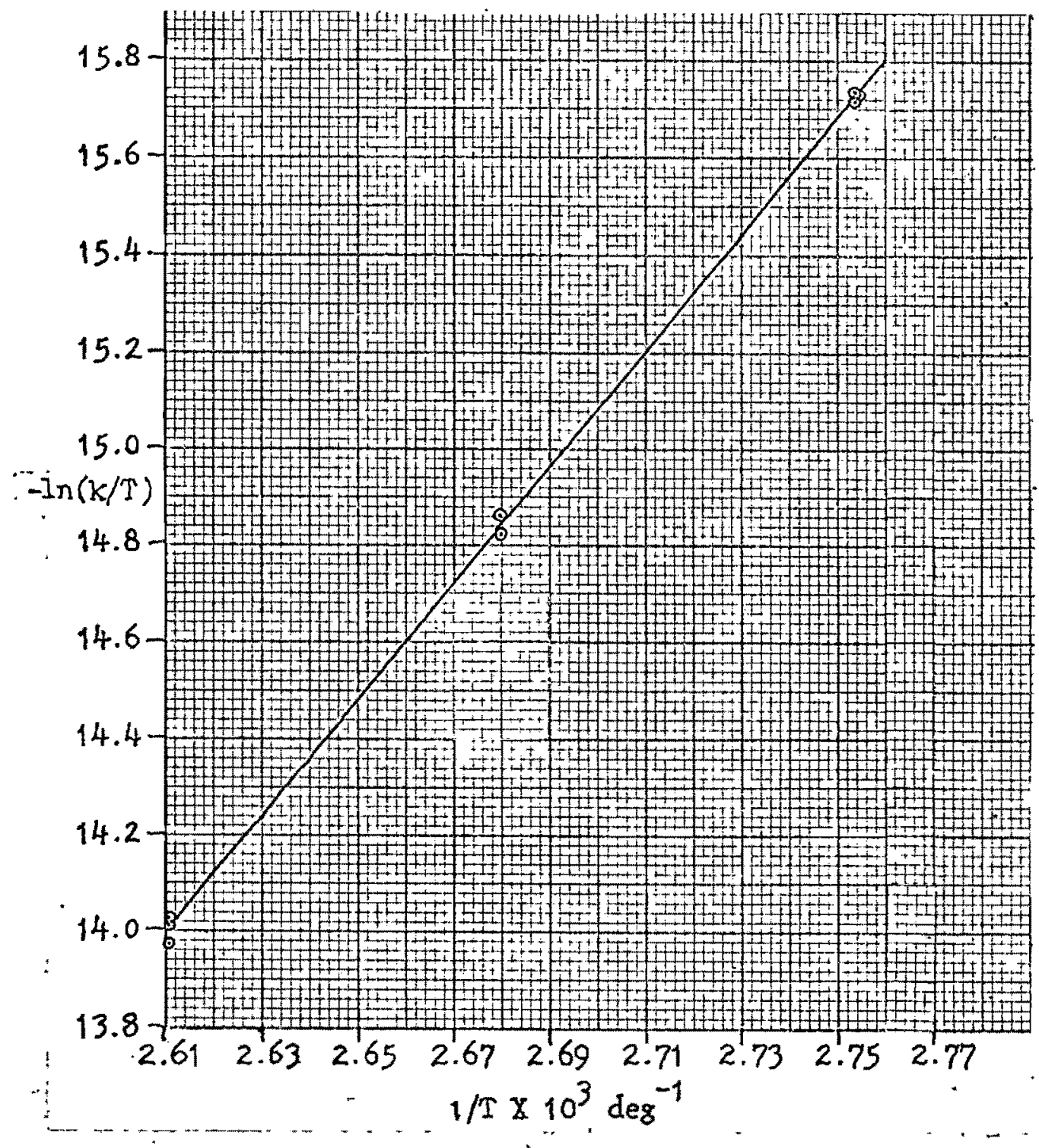

Figure 2: Arrhenius plot of. In $(k / T)$ versus $1 / \mathrm{T}$ employing rate constants calculated from data collected up to $98 \%$ reaction. 


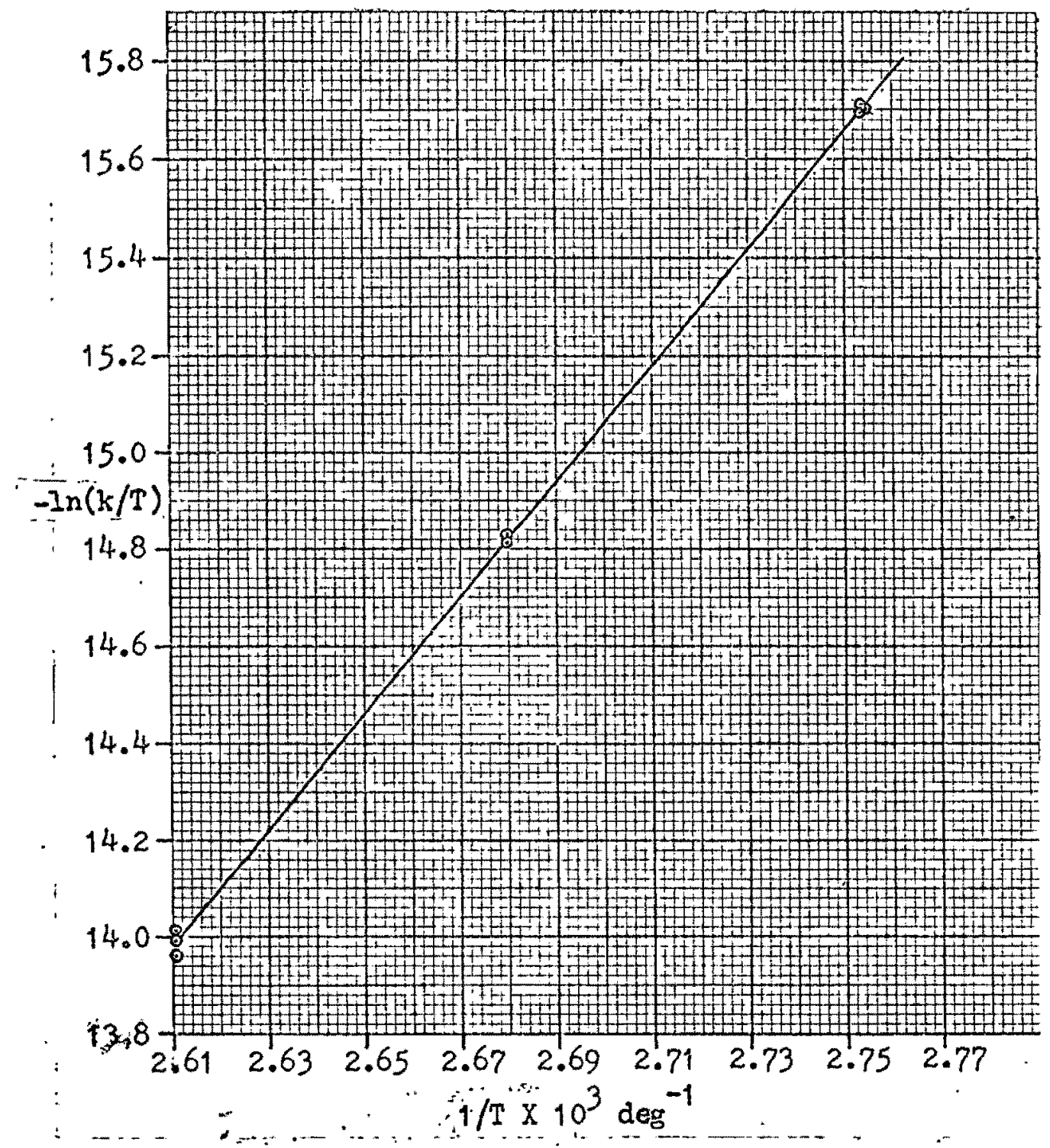

Figure 10. Arrhenius plot of $-\ln (\mathrm{k} / \mathrm{T})$ versus $1 / \mathrm{T}$ employing rate constants calculated from data collected up to $85 \%$ reaction. 


\section{SUMMARY OF ACTIVATION PARAMETERS \\ FOR REARRANGEMENT OF dl-DIENE \\ AND RELATED REACTIONS}

Compound

rearranged.

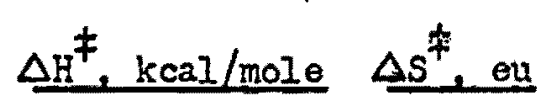

$\underline{\text { Ref. }}$

Diethyl allyl-

28.4

$-13.5$

20

malonate

Bullvalene
trans,trans-2,8-
trans-Bicyclo $[8.4 .0]$ -
tetradecadiene
1-Hexene-5-yne-4-ol
1,1-Dideuteriohexa
$1,5-d i e n \theta$
meso-3,4-Dimethyl-
hexa-1,5-diene
dl-3,4-Dimethyl-
hexa-1,5-diene
$" \quad$ " $\quad$
dl-3,4-Diphonyl-
hexa-1,5-diene

$11.6^{2}$

$-$

21

25

22

$30^{a}$

$-14$

23

33.5

$-13.8$

17

$33.1, \cdot-11.2$

24

$35: 2^{b}$

$-5.9$

It

$35.3^{c}$

$-10.1$

n

$23.8^{d} \quad-12.9$

this work

" "

$23.9^{\circ}-12.6$

n

${ }^{a}$ Only the Arrhenius activation energy was reported. $\mathrm{b}$

For rearrangement to trans,trans-octa-2,6-diene.

$c$

For rearrangement to cis, cis-octa-2,6-diene

d.

Evaluated from rate constants which had been determined from data collocted up to $85 \%$ reaction.

e

Evaluated from rate constants which had been determined from data collectod up to $98 \%$ reaction. 


\section{ALUNINA-CATALYZED REARRANGEMENT}

Comparatively fow examples of catalyzed Cope and Claison rearrangements are known. Nost involve the use of transition-metal complexes as the catalytic agents. For example cls,trans-1,5cyclodecadiene (XIV) was isomerized essentially quantitatively in 6-7 days at room temperature to cis-divinylcyclohexane (XV) by means of bis-benzonitrile-palladium(II) chloride or sodium chloroplatinite in benzene (25). In addition Heimbach and Brenner (26) observed a<smiles>C1=C\CCC/C=C/CCC/1</smiles>
XIV

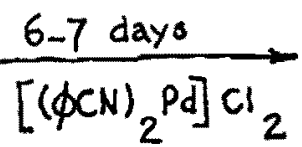
room temp<smiles>C=CC=C1CCCCC1C=C</smiles>

$\mathrm{Xx}$
$99 \%$

catalyzed Cope rearrangement during preparation of cis-1,2-divinylcyclobutane by dimerization of liquid butadiene with a $1: 1$ mixture of bis-(trans,trans-cycloocta-1,5-diene) nickel and tris-(2-biphenyl) phosphite. Under the reaction condition employed the desirod product was found to undergo isomerization to cycloocta-1,5-diene, the same compound generated by its purely thermal rearrangement.

A somewhat similar reaction, the Claisen rearrangement of 2 allyloxypyridine (XVI) to 1-allyl-2-pyridone (XVII), was brought about
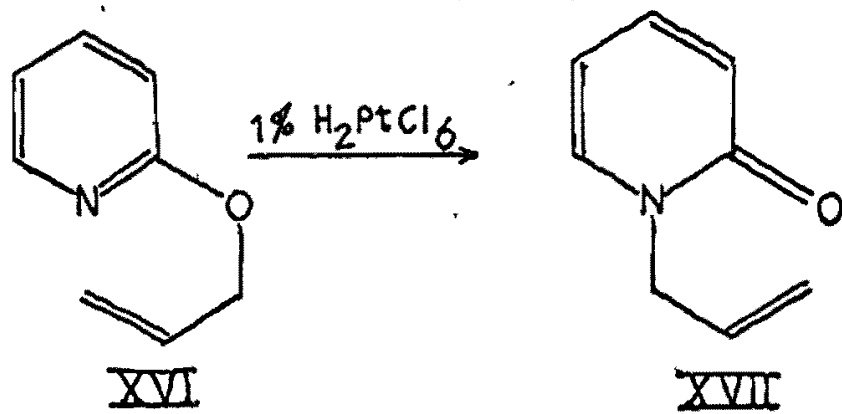
with aid of $1 \%$ of chloroplatinic acid $\left(\mathrm{H}_{2} \mathrm{PtCl}_{6}\right)$, sodium chloroplatinite $\left(\mathrm{Na}_{2} \mathrm{PtCl}_{4}\right)$ boron trifluoride-etherate $\left(\mathrm{BF}_{3} \cdot \mathrm{O}\left(\mathrm{C}_{2} \mathrm{H}_{5}\right)_{2}\right)$ or stannic chloride (27). Decreased conversions were obtained by use of palladiumoncarbon (55\%), and aluminum chioride, nickel(II) chloride, platinum black or alumina each of which gave less than $15 \%$ conversion. The failure of the latter substances to behave as effective catalysts was attributed in part to their low solubility in the reaction medium. Subsequent investigations revealed that the only products obtained from boron trifluoride-etherate-catalyzed isomerizations were those characteristic of the abnormal Claisen rearrangement.

In the course of this study it was observed that a sample of contaminated dl-3,4-diphenylhexa-1,5-diene, when chromatographed on very active noutral alumina (Woelm grade super. I), rearranged to transtrans-1,6-diphenylhexa-1,5-diene. Previous chromatographic puriftcations carried out on alumina of somewhat lower activity (e.g. Woelm grade I), including acid-washed alumina, failed to give rearrangement. Needless to say these results were not only unusual but 2 so completely unexpected and were assumed initially to be artifactual. However further investigation revealed that rearrangement did in fact occur, and was not the result of impurities in the diene. The rearrangement, when carried out using a 100:1 ratio of alumina to diene, gave an approximately $50 \%$ conversion to trans, trans isomer (the only product observed) after a 2-hr period. Attempts to effect a similar rearrangement of meso-3.4-diphenylhexa-1,5-diene wore also successful. Chromatography of the meso-diene on very active alumina using, a 100:1 ratio of adsorbent to olefin gave, after $70 \mathrm{hr}, \mathrm{a} 15 \%$ conversion. The rearrangement 
product consisted of cis, trans and trans,trans-1,6-diphenylhexa-1,5diene in the ratio of $9: 1$ based upon glpc analysis. The latter was complicated by the thermal rearrangement of residual meso-diene on the glpc colum during analysis. (Compare Fig. 15-17 in the experimental section.) However, moderately reliable corrections were made for its contributions to the total amounts of cis,trans and trans, trans isomers present. It is apparent from the observed product distributions that both of these alumina-catalyzed rearrangements parallel the thermally induced reactions. The absence of large amounts of side products predicted to result from radical or ionic reactions (e.g.trans-1,4diphenylhexa-1,5-diene) may indicate that dissociation to these intermediates does not occur to a significant degree. These reactions aro clearly deserving of additional investigation. 


\section{EXPERDMENTAL}

Instmumentation and Apparatus. Infrared analyses were carried out on either Perkin-Elmer Model 137, "Infracord" or Perkin-Elmer Model 467 spectrophotometers.

- MMR spectra were aquired with the aid of a Varian Associates A-60 $(60 \mathrm{MHz}$ ) analytical nmr spectrometer. Tetramethylsilane was used as an Internal standard.

Uitraviolet measurements were made on a Cary 14 recording spectrophotometer (manufactured by Cary Instruments) with a limit of accuracy of \pm 0.002 absorbance units in the $0-1$ absorbance range and \pm 0.005 units near an absorbance of 2. Samples and reference solvents were contained within a matched pair of 1-cm quartz cuvettes. A specially designod wash-bottlo, shown below in Fig. 11, was constructed to facilitato: rapid, effective cleaning of the cuvettes. Approximately 10 openings, each less tha $0.5 \mathrm{~mm}$ in diameter, were cut into the closed end of the siphon sidearm by means of a small "sand-blasting" unit, providing tho apparatus with a spray head capable of directing fine, forceful streams of solvent onto all of the cuvette"s interior surfaces while at the same time minimizing solvent consumption. The U-shaped trap desig.. nated $A$ in the figure was constructed such that solvent would neither siphon into the reservoir when the latter was nearly empty nor drain througi the spray head when the reservoir was filled to the level of the gas inlet. A cuvette was cleaned by placing it over the spray head, applying a slight gas pressure (ca 2 psig) to the solvent in the reservoir by means of the sidemam and slowly raising and lowering the cuvette to assure uniform distribution of solvent over its inner walls. After 3-5 seconds the cuvette was drained by tapping gently on absorbent, 


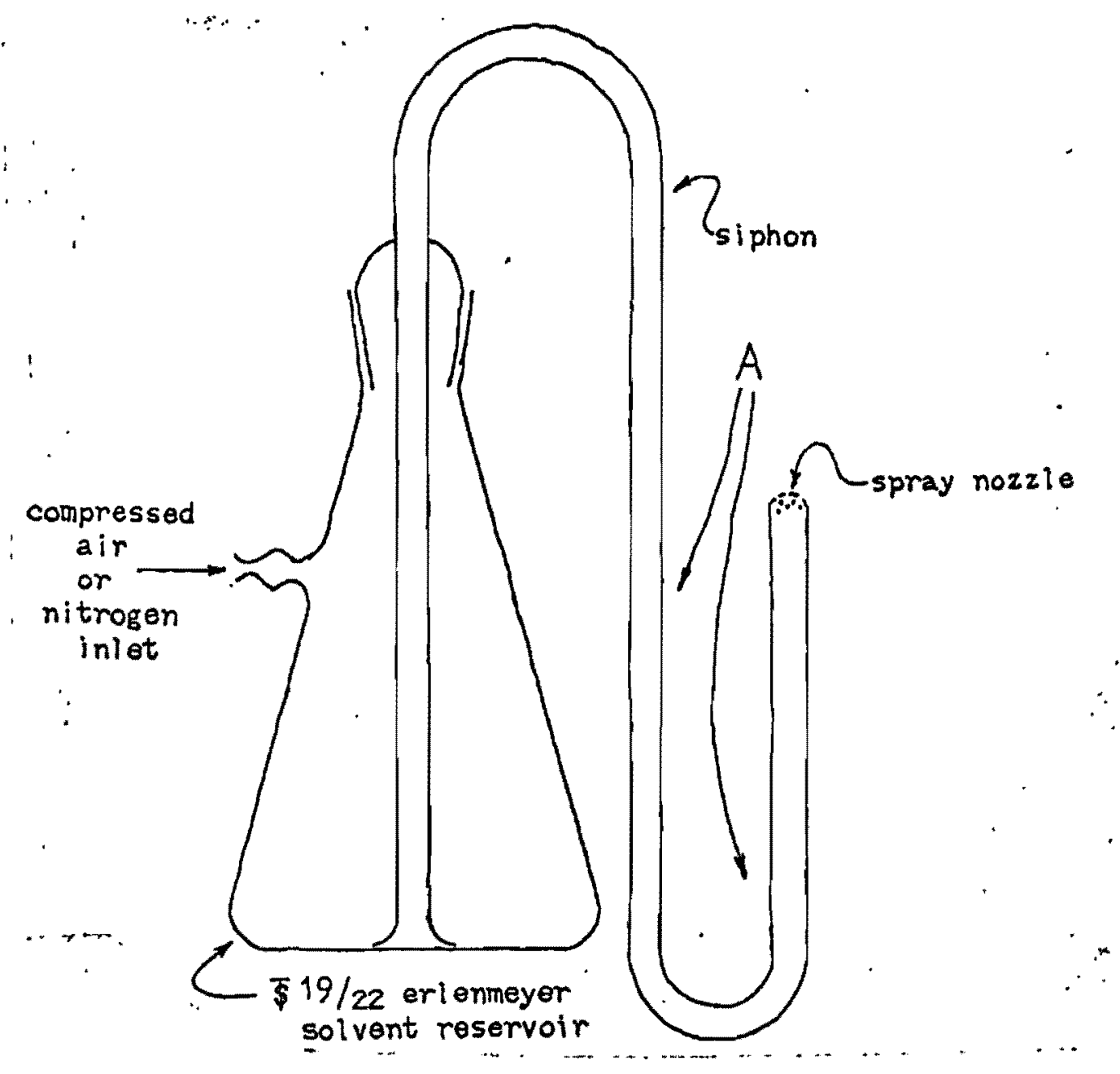

Figure 11. Cuvette wash-bottle.

Iint-free paper and dried by means of a jet of nitrogen or compressed air.

Gas-liquid-partition chromatographic (glpc) analyses of meso- and dl-3,4-diphenylhexa-1,5-dienes and their rearranigement products were carried out with a Hewlett-Packard Kodel 5750B Research Chromatograph equipped with dual flame-ionization detectors and a disc integrator. Seperations were effected upon $1 / 8^{\prime \prime} \times 8^{\prime}$ stainless steel columns packed with 5\% XE-60 cyanosilicone gum rubber on 80-100 mesh acid-washed silanized Chromasorb W. Compositions of chromatographic samples were calculated from relative peak areas. 
Melting points were determined with a Thomas-Hoover capillary melting point apparatus of the stirred-fluid-bath type (manufactured by Arthur H. Thomas Co.) AII melting points are uncorrected.

A rotary evaporator operating at water aspirator vacuum was employed to evaporate solvents.

Moisture-sensitive reagents, such as potassium tert-butoxide and dimethyl sulfoxide were manipulated under an anhydrous nitrogen atmosphere either in a glove bag or glove box. The former apparatus is available from Instruments for Research and Industry, the latter from Irabconco Corp.

Solvents. DMSO (Dimethyl sulfoxide, Mallinckrodt analytical reagert grade) was purified by vacuum fractionation at $20-30 \mathrm{~mm}\left(\mathrm{l}_{2}^{\prime}\right)$ from either calcium hydride $\left(1: 100 \mathrm{w} / \mathrm{w}\right.$ ) and calcined (at $400^{\circ}$ for $2 \mathrm{hr}$ ) Linde $13 X$ molecular sieves $(1: 10 \mathrm{w} / \mathrm{w})$, or from sodium hydride $(1: 1 \mathrm{co}: .$. w/w, from a $50 \%$ oil dispersion freed of mulling oil by rapeated washing with pentane followed by drying in vacuo). Although the latter method is more rapid and, generally, less troublesome it suffers from one particular disadvantage in that the sodium hydride-DMSO mixture is potentially explosive. The distillation employed a $2 \mathrm{~cm} \times 70 \mathrm{~cm}$ vacuumjacketed silvered column packed with .050" X .100" X .100" Monel metal helices (by Podbielniak). A substantial amount of DMSO (up to 30\%) was distilled as an often discolored fore-run. Approximately 70-80\% of the remainder was collected as pure material (bp range less than $\left.0.5^{\circ}\right)$. The receivers, in which the distillate was stored until use, were detached from the still (under positive tank-nitrogen pressure) and sealed quickly with silicone-greased glass stoppers to prevent 
absorption of atmospheric moisture.

n-Heptane (MaIlinckrodt SpectrAR spectrophetometric grade) was

used in all but the column chromatographic experiments for which

Mallinckrodt Analytical Reagent grade heptane was satisfactory. The

former was recovered by fractionation through the column describod $\because$ above.

Unless otherwise specified, solvents other than these mentioned above were of reagent quality and were used without further purification:

Synthesis of Starting Materials

Dimethyl meso- and de- $\beta, \gamma$-Diphenyladipates. The method of synthesis was essentially that employed by Oommen and Vogel (28), as modified by Lutz (14). Methyl trans-cinnamate (151, g, 0.93 mole, vacuum fractionated through a 12 "Vigreux column) was reductively coupled in moist ether over aluminumamalgam prepared from $253 \mathrm{~g}$ ( 9.4 moles) of aluminum. The nearly uncontrollably exothermic reaction yielded $25.0 \mathrm{~g}$ of mesoester and $38.0 \mathrm{~g}$ of impure dl-ester which was subsequently reduced. The total yield of adipates was $63.0 \mathrm{~g}$ (41\%).

It should be noted that even modest separation of the meso- and dl-3,4-diphenylhexane-1,6-diols obtained by hydride reduction of the impure dl-ester mentioned above was :effected only with 'difficulty.. : Consequently it is imperative that the reduction be carried out using pure starting materials.

dl-3,4-Diphenylhexane-1,6-diol. Iithium aluminum hydride $(7.0 \mathrm{~g}$. $0.185 \mathrm{~mole}$ ) was added in one portion" to anhydrous ether ( $340 \mathrm{ml}$ ) 
contained in a two-liter three-neck flask fitted with a nagnetic stirrer, mercury pressuremelief valve and powder funnel. A mixture of dimethyl meso- and dl- $\beta, \gamma$-diphenyladipates $(19.9 \mathrm{~g}, 0.0610$ mole, mp 66-95 $)$ was added to the stirred suspension of hydride, (which boiled briefly as a result of the heat liberated), followed by $490 \mathrm{ml}$ more anhydrous ether. Finally $15.1 \mathrm{~g}(0.0463 \mathrm{~mole})$ more of dl-meso-adipate mixture in $150 \mathrm{ml}$. of anhydrous ether, followed by an additional 300-ml portion of anhydrous ether, were introduced. The flask was then stoppered and the mixture stirred for $36 \mathrm{hr}$.

Water (809 $\mathrm{ml}$ ) was added cautiously to the stirred grey suspension until there was no further evidence of hydrogen evolution. A greyish precipitate slowly formed, from which the supernatant liquid was decanted. After pressure filtration to remove suspended particulate matter the decantate was stripped of solvent and the resulting residue dried on full oil-pump vacuum to yield $14.5 \mathrm{~g}$ of a gum. The grey precipitate was washed with two portions of chloroform ( $500 \mathrm{ml}, 200 \mathrm{ml}$ ) and filtered through first a coarse and then a fine sintered glass funnel to remove last traces of solid. The filtrate was concentrated at reduced pressure to $6.75 \mathrm{~g}$ of a gum. Addition of more water to the grey inorganic residue resulted in liberation of heat and the formation of a viscous yellow gel which was triturated with chloroform and dichloromethane. The supernatants were separated by suction filtration. After separation from the aqueous phase the organic layer was concentrated and dried at oil-pump vacuumi to give a further $2.67 \mathrm{~g}$ of gummy residue. Finally the mixture of aluminum salts was dissolved in aqueous sulfuric acid (200 mi water $+50 \mathrm{ml}$ 6N sulfuric acid) and 
extracted with chloroform $(3 \times 65 \mathrm{ml})$. Evaporation under vacuum of the dried $\left(\mathrm{NgSO}_{4}\right)$ extracts yielded $2.23 \mathrm{~g}$ more gum.

The viscous mixtures of meso- and d]-3.4-diphenylhexane-1,6-diols, totaling $26.1 \mathrm{~g}$, were combined and fractionally crystallized with difficulty from benzene $(75 \mathrm{ml}$ ) to give $6.63 \mathrm{~g}$ of impure dl-diol, $\mathrm{mp}$ $99-128^{\circ}$. The filtrate from the first crop was diluted to $200 \mathrm{ml}$ with benzene, seeded with dl-diol, and placed in a cold room at $6^{\circ}$ to crystallize. After 36 hours a second crop of dl-alcohol $(4.56 \mathrm{~g}$, mp$\left.100-130^{\circ}\right)$ was collected by suction filtration. A third crop of tire dlalcohol $(1,61 \mathrm{~g}$, mp 98-132\%) was obtained by concentrating the filtrate from the second crop to $125 \mathrm{ml}$, seeding with dl-alcohol at ambient tomperature, and chilling at $6^{\circ}$ for 13 hours. Attempts to

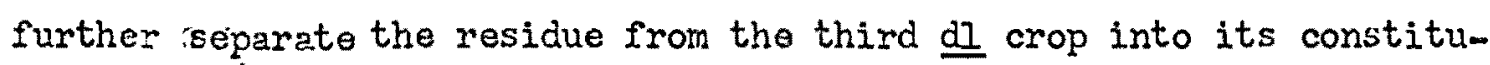
ents by fractional crystallization of either dl-diol (from benzene) or meso-alcohol (from benzene or tetrahydrofuran) failed. Resolution by chrometography over Woelm Super I neutral alumina also proved unsuccessfuI.

The three crops of impure dl-2lcohol were combined with $1.62 \mathrm{~g}$ of material from $\ddot{z}$ previous reduction ( $14.4 \dot{\mathrm{g}}$ total) and recrystallized first from benzene and then from ethanol-water $(2: 1 \mathrm{v} / \mathrm{v})$ to give $4.02 \mathrm{~g}$ of di-diol, mp 99-107. Evaporation to drymess in vacuum of the ethanolic filtrate gave $5.25 \mathrm{~g}$ of material which when recrystallized again from benzene yielded another crop of dl-alcohol (3.82 g, mp ; ; 99-102 ${ }^{\circ}$ ). The $4.02 \mathrm{~g}$ dl-diol crop was recrystallized from benzene and from $2: 1 \mathrm{v} / \mathrm{v}$ ethanol-water to give $2.39 \mathrm{~g}$ of dl-alcohol with groatly broadened relting range (mp $98.5-140^{\circ}$ ). A portion of this crop was to 
have been examined by IR to deternine, if possible, the cause for the enlarged melting range. Iruing preparation of the IR sample in chloroform it was noted that a precipitate formed, which was subsequently identified by. melting point as meso-diol (mp 1705-176 , lit. for meso $178.5-179^{\circ}(14)$. This observation was put to practical use in the purification of the remaining impure dl-diol from the $2.39 \mathrm{~g}$ crop. The latter was triturated with chloroform, filtered, and tho filtrate ovaporated in vacuum to yield $1.97 \mathrm{~g}$ of dl-alcohol, mp $99.7-102.5^{\circ}$. (1it. mp $99-100^{\circ}(14), 103.6-104.7^{\circ}$ (29). This crop was combined with another ( $3.82 \mathrm{~g} \mathrm{mp} 99-102^{\circ}$ from benzene as described above) and used in the tosylation reaction which follows.

Cl-3,4-Diphenylhexamethylene $\mathrm{D}$ - Toluenesulfonate. The ester was prepared by the method of Lutz (14) from dl-diol $\left(5.79 \mathrm{~g}, \mathrm{mp} 99-102^{\circ}\right)$ and p-toluenssulfonyl chloride $\left(11.0 \mathrm{~g}\right.$, mp $66 \ldots 69^{\circ}$, Iit. $67.5-69^{\circ}$ (14), recrystallized from carbon tetrachloride). The crude tosylate $(10.3 \mathrm{~g}$, mp $93.5-105^{\circ}$ ) was recrystallized from carbon tetrachloride; filtration and washing were carried out at $-25^{\circ}$. The final yield of ester was $7.82 \mathrm{~g}(63 \%)$, mp $97-99^{\circ}$ (1it. $99.5-101.5^{\circ}$ (14.).

meso-1,6-Dibromo-3,4-diphenylhexane. Lithium bromide $(7.32 \mathrm{~g}$, $0.0843 \mathrm{~mole}$ ) was rapidly weighed up in a stoppered $250 \mathrm{ml}$ round-bottom flask fitted with a magnetic stirrer. meso-3,4-Diphenylhexamethylene p-toluenesulfonate $(12.1 \mathrm{~g} 0.0209$ mole $)$ was rapidly introcluced and the flask sealed with a serum cap. Reagent acetone $(75 \mathrm{ml}$ ) was injected into the mixture of halide and ester. A reflux condenser with drying tube was attached to the reaction flask, and the contents were stirred magnetically and refluxed $12 \mathrm{hr}$. (A shorter reaction period might have 
given comparable conversion.) After cooling to room temperature, solvent was cerefully removed at aspirator vacuum and the residue dried, with the aid of a warm vater bath, at full oil-pump vacuum for $2.5 \mathrm{hr}$. The residue was triturated with $200 \mathrm{ml}$ of water, the resulting slurry suction filtered, and the crude meso-1,6-dibromo-3,4-diphenylhexane washed ith six 10-ml portions of water. The air-dried product $(7.72 \mathrm{~g}$, mp 144.5-145 ${ }^{\circ}$, lit. $144-144.5^{\circ}$ (14) represented a $94 \%$ yield.

This synthetic procedure was included as it represented an improvement in the preparation of the meso-bromide as described by Lutz (14). Apparently moisture is necessary for the bromination reaction to proceed to completion.

dl-1,6-Dibromo-3,4-diphenylhexane. dl-3,4-Diphenylhexamethylene p-toluenesulfonate $(15.2 \mathrm{~g}, 0.0262 \mathrm{~mole})$ and lithium bromide $(0.15 \mathrm{~g}$, 0.105 mole) were veighed up in a stoppered $250 \mathrm{ml}$ round-bottom flask fitted with a magnotic stirring bar. Reagent acetone ( $92 \mathrm{mI}$ ) was injected into the reaction flask through a serum cap. A reflux condenser with drying tube was attached to the flask, and the contents stirred magnetically. Within $10 \mathrm{~min}$. the mixture became homogeneous, and was then gently refífuxed for $2 \mathrm{hr}$. Solvent was carefully (to prevent bumping) evaporated at aspirator vacuum, and the residue dried at full oil-pump vacuum $1 \mathrm{hr}$. The dried residue was triturated with water (140 mi) and the resulting slurry suction filtered. The crude dlbromide was then washed with four $30 \mathrm{ml}$ portions of water, aspirated as dry as possible, and finally dried over phosphorous pentoxide $36 \mathrm{hr}$

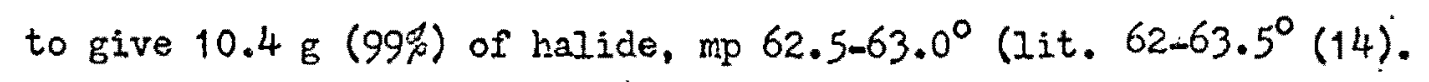


meso-3,4-Diphenylhexa-1,5-diene. (Run 1) A solution of potassium tert-butoxide was prepared by dissolving, with the aid of magnetic stirring, $408 \mathrm{mg}$ of the base in DMSO $(5.9 \mathrm{ml})$, and pressure fijtering $\left(\mathrm{N}_{2}\right)$ the mixture to remove insoluble matter. The filtrate was collect. ed in a 10-ml flask which was subsequently fitted vith a serum cap to exclude moisture. A $1 \mathrm{ml}$ aliquot was removed by syringe, diluted with deionized wator $(20 \mathrm{ml})$ and titrated with standardized $0.1 \mathrm{~N}$ hydrochloric acid to an alizarin end-point. The calculated concentration of the base solution was $0.220 \mathrm{~N}$. A halide solution was prepared by magnetical1.y stirring together, for $0.5 \mathrm{hr}$, meso-1,6-dibromo-3.4-diphenglhexane (120 mg, $0.303 \mathrm{mmole}$ ) and $9.6 \mathrm{ml}$ of DMSO, to which $2.80 \mathrm{ml}$ of $0.220 \mathrm{~N}$ potassium tert-butoxide in DMSO was added continuously over a perioc of $90 . \mathrm{sec}$. After an additional $50 \mathrm{sec}$. the solution was poured over $60 \mathrm{~g}$ of ice in a separatory funnel, swirled briefly, and neutralized to a phenolphthalein end-point with $18 \%$ hydrochloric acid. The cold mixture was shaken three minutes (shaking appeared to promote formation of a crystalline product) let warm 'till the ice melted and suction filtered. The crude meso-3,4-diphenylhexa-1,5-diene was washed with cold watep (five 10-20 -ml portions), dxied by aspiration and then at full oil-pump vacuum for $0.5 \mathrm{hr}$. The crude diene had mp 65-83.5 . The presence of small amounts of ether and another side product (possibIy a rearranged olefin), together with unreacted meso-bromide, was indicateal by IR and NMR. Crude yield, (72 $\mathrm{mg}$; theoretical yield - $71 \mathrm{mg}$ ) (Run 2) A standard solution of potassium tert-butoxide was prepared by shaking together $8.50 \mathrm{~g}(0.076 \mathrm{~mole})$ of the base and $140 \mathrm{ml}$ of DMSO, and pressure filtering $\left(\mathrm{N}_{2}\right)$ the mixture to separate insoluble 
matter. A $118 \mathrm{ml}$ portion of this solution $(0.162 \mathrm{~N}$, standardized as described, above) was injected continuously over a 20 . min period into a solution of $4.96 \mathrm{~g}(0.0125 \mathrm{~mole})$ of meso-1,6-dibromo-3, 4odiphenylhexane in $400 \mathrm{ml}$ of DMSO. After an additional 10 min period the solution was poured over $1 \mathrm{~kg}$ of jce and worked up as described above to give $1.71 \mathrm{~g}$ of crude meso-diene, mp $67-80^{\circ}$. An additional $1.43 \mathrm{~g}$ of diene, obtained from chloroform extracts of the aqueous DMSO filtrate, was chromatographed over $150 \mathrm{f}$ of Woelm grade I basic aIumina. Elution with hexane gave $577 \mathrm{mg}$ of meso-diene, mp $55-74^{\circ}$, which was combined with that aquired by filtration and recrystallized from ethanol (95\%). Two crops,

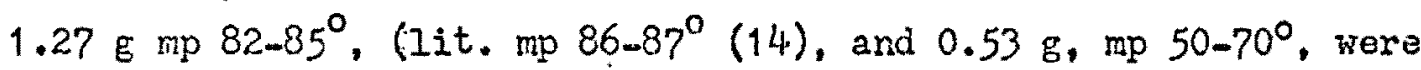
obtaired. The latter, found by IR to be rich in unreacted meso-bromide, was not further purified. Consumption of alkoxide by reaction with atmospharic moisture and carbon dioxide during the course of preparation and uso of the base solution may account for the absence of complete elimination observed here. This is reasonable since few (probably ineffective) precautions were taken to prevent absorption of moisture. (Neither this reaction nor the one preceding it were carried out in an anhydrous atmosphere.)

Attempted preparation of meso-3,4-diphenylhexa-1,5-diene (accompaied by product rearrangement). meso-16-Dibromo-3,4-diphenylhexane (101 $\mathrm{mg}, 0.255 \mathrm{mmole}$ ) and potassium tert-butoxide (134 mg, $1.19 \mathrm{mmole}$ ) were combined rapidly in a nitrogen-filled 50-ml round-bottom flask equipped with a magnetic stirring bar and glass stopper. DMSO (5 ml) was rapidly introduced in one portion with stirring into the mixture of solid reactants, the stopper wäs reattached to oxclude moisture 
and stirring was continued. After $0.5 \mathrm{hr}$ the reaction mixture was poured into $35 \mathrm{ml}$ of cold $\left(0^{\circ}\right)$ saturated aqueous sodium chloride solution, shaken briefly and noutralized to a phenolphthalein end-point. with 18\% hydrochloric acid. Upon warming to room temperature a small amount of solid present melted. The mixture was then chilled in ice to cause the oil to resolidify, the crystalline deposits which formed were separated by decantation, and taken up in chloroform. Evaporation in vacuum of the chloroform solution gave $70 \mathrm{mg}$ of oil which contained no detectable (by $M M R$ ) amount of meso-diene. The material was not further characterized. An WMR spectrum of the crude product is given in Figure 14.

Attempted base-catalyzed isomerization of meso-3,4-diphenylhexa1.5-diene. Potassium tert-butoxide $(0.371 \mathrm{~g})$ was rapidly veighed in a glass-stoppered $5-\mathrm{ml}$ round-bottom flask equipped with a magnetic stirring bar. Dried (molecular sieves) DMSO $(1.67 \mathrm{ml}$ ) was injected into the flask and the mixture was vigorously stirred for five minutes, giving a slightly turbid solution. tert-Butyl alcohol (1.67 ml) followed by meso-3,4-diphenylhexa-1,5-diene (102 mg, 0,435 mole), were thon introduces. Stiring was continued $0.5 \mathrm{hr}$, after which the turbid re. action mixture was poured over about $20 \mathrm{~g}$ of ice in a separatory funnel, shaken briefly and neutralized to a phenolphthalein end-point with $18 \%$ hydrochloric acid. The mixture was extracted with two $2-\mathrm{ml}$ portions of dichloromethane which were combined, washed twice with water (20mi total), dried over anhydrous magnesium sulfate and evaporated to dryness in vacuum. IR and $M M R$ spectra of the residue revealed that presence of meso-diene only, A slight depression of the melting point from 
$86.3-87.3^{\circ}$, for the untreated meso-dione, to $79.5-83.5^{\circ}$ was observed for the recovered meso. It was therefore concluded that no rearrangement had taken place.

$$
\text { dl-3,4-Diphenylhexa-1,5-diene. Potassium tert-butoxide }(1.61 \mathrm{~g} \text {, }
$$
$80 \%$ by weight, 0.0115 mole) was weighed up into a $50 \mathrm{~min}$ round.bottom flask fitted with a magnetic stirring bar and a glass stopper. DMSO (38 $\mathrm{ml}$ ) was introduced and the mixturo was stirred until the alkoxide dissolved (about $1 \mathrm{~min}$ ) leaving a yellow-orange solution containing some undissolved solid. dI-1,6mibromom 3,4-diphenylhexane $(2.128,5.35$ mole) was introduced with stirring. The reaction vessel was stoppered with a silicone-greased glass stopper and the mixture allowed to stir for $50 \mathrm{~min}$. Thereafter the reaction mixture was poured into $120 \mathrm{ml}$ of cold water, neutralized to a phenolphthalein end-point with $2 \%$ hydrochloric acid and extracted with pentane $(3 \times 90 \mathrm{mI})$. The pentane extracts were combined, washed with water $(2 \times 100 \mathrm{ml})$, dried over anhyd. rous magnesium sulfate and evaporated in vacuum. The residue was treated with several portions of carbon tetrachloride which were evaporated by oil-pump in order to remove residual DMSO from the crude product.

The oil $(1.29 \mathrm{~g})$ which was obtained was combined with another $1.20 \mathrm{~g}$ of crude dl-diene from a similar elimination. A portion of the crude diene $(2.13 \mathrm{~g})$ was purified by dry-column chromatography (30) On $340 \mathrm{~g}$ of Woelm grade-III (dry-column grade) silica gel (containing $0.5 \%$ inorganic muorescent indicator). The column was developed with hexane, the progress of the separation being followed with short-wave UV light (the diene fluoresces dark blue). dl-Diene was leeched.from the second quarter-section of the column by successive triturations 
with dichloromethane followed by pressure filtration. (More than 98\% . of the diene racovered was obtained from the first three triturations. The remainder was acquired by extraction of the adsorbent with dichm loromethane in a Soxhlet extractor). The combined dichloromethane extracts were evaporated to dryness in vacuum to yield $1.05 \mathrm{~g}$ of dl-diene, mp $33-35^{\circ}$, which vas recrystallized twice from $95 \%$ ethanol (with filtration and washing being carried out in a freezer at $-13^{\circ}$ ) to give $381^{\circ} \mathrm{mg}$ of dl-diene, $\mathrm{mp} 35.0-35.3^{\circ}$ (lit. $35.1-35.4^{\circ}$ (14). dl-Diene $\left(169 \mathrm{mg}, \mathrm{mp} 28-33^{\circ}\right)$ from a small-scale dry-colum chromatographic purification was combined with the filtrate from the first recrystallization of the main sample, and a second crop (219 mg, mp $\left.32.5-34.2^{\circ}\right)$ was collected. The combined crops represented a yield of $25 \%$.

Kinetics of the thermal rearrangement of d1-3,4-diphenylhexa-1,5-diene

$$
\text { Constant temperature bath. A 15-1 Pyrex glass jar containing }
$$

10 I of Lauda UItra-Therm $330 S$ bath fluid (catalogue number 2757 030-5. distributed by Brinkmann Instruments) served as a constant temperature bath. The bath was equipped with a lightnin' Model I stirrer (manufacm tured by Mixinğ Equipment Co. Inc., Rochester, N.Y., and marketed by Scientisic Glass Apparatus Co. Jand a Precision Scientific Co. electronic relay (catalogue number 62690) to which a mercury thermoregulator (Philadelphia Micro Set differential-range typo) was connected. Temperatures were determined by means of two mexury-in-glass thermometers reading from 75 to $105^{\circ}$ (in 0.1 -degree units) and from $95-155^{\circ}$ (in 0.2-degreo units). Both were standardized against a pair of thermometers calibrated by the Bureau of Standards (see Appendix A). 
A Philadelphia differential themnometer was employed to determine the reproduciblity of the bath's temperature over several runs as well as to monitor temperature fluctuations during the course of a single run. All five of the thermometers were obtained from Scientific Glass Apparatus Co.

Sample preparation. Ampules having 16-cm $\times 4$ mm necks and 15-mm (od) X 35-m bulbs ware fabricated from Pyrex glass tubing. Fourteen such ampules wero nomally employed in a kinetics mun. Prior to introduction of a sample each ampule ras rinsed twice by being partially filled rith heptano, shaken briefly, drained and dried by evacuation using the apparatuses show (Fig. 12 and 13 respectively). (Draining was accomplished simply by placing the ampule in the position shown and applying suction to the filtor flask.)

A solution of ca $5 \times 10^{-5} \mathrm{M}$ dl-3,4-diphenylhexa-1,5-diene in heptane was jropared by dissolving 114-117 mg (weighed to the nearest $0.1 \mathrm{mg}$ ) of dl-diene in heptane, diluting to $100 \mathrm{ml}$, withdrawing a $1 \mathrm{ml}$ aliquot of this solution and diluting again to $100 \mathrm{ml}$. (Volumetric apparatus used were calibrated with heptane.) Into each of the dried ampules, through a capillary pipet which reached to the bottom of the sample chamber, was placed a 2-3 $\mathrm{ml}$ aliquot of the dilute dl-diene solution. The ampules were then randomly attached to the evacuator shown in ( $\mathrm{Pig}$. 13) chilled to $-78^{\circ}$ for at least five minutes and finally evacueted at oil-pump vacuum (to less than $0.6 \mathrm{~mm}$ ) for five minutes or more. Thereafter the ampules were flame sealed (with the upper portions of the necks remaining attached to serve as handles). 


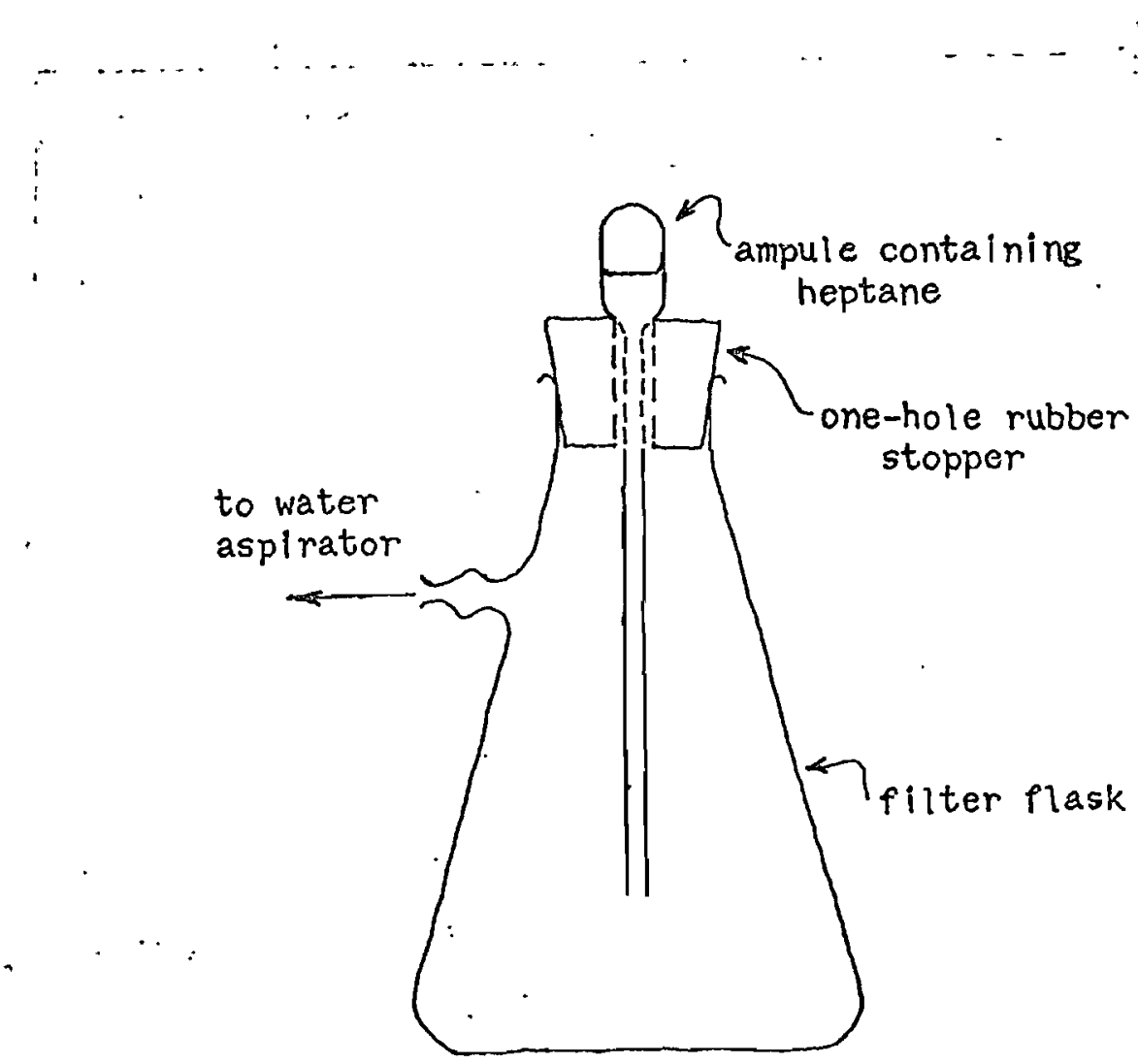

Figure 12. Apparatus for draining ampules.

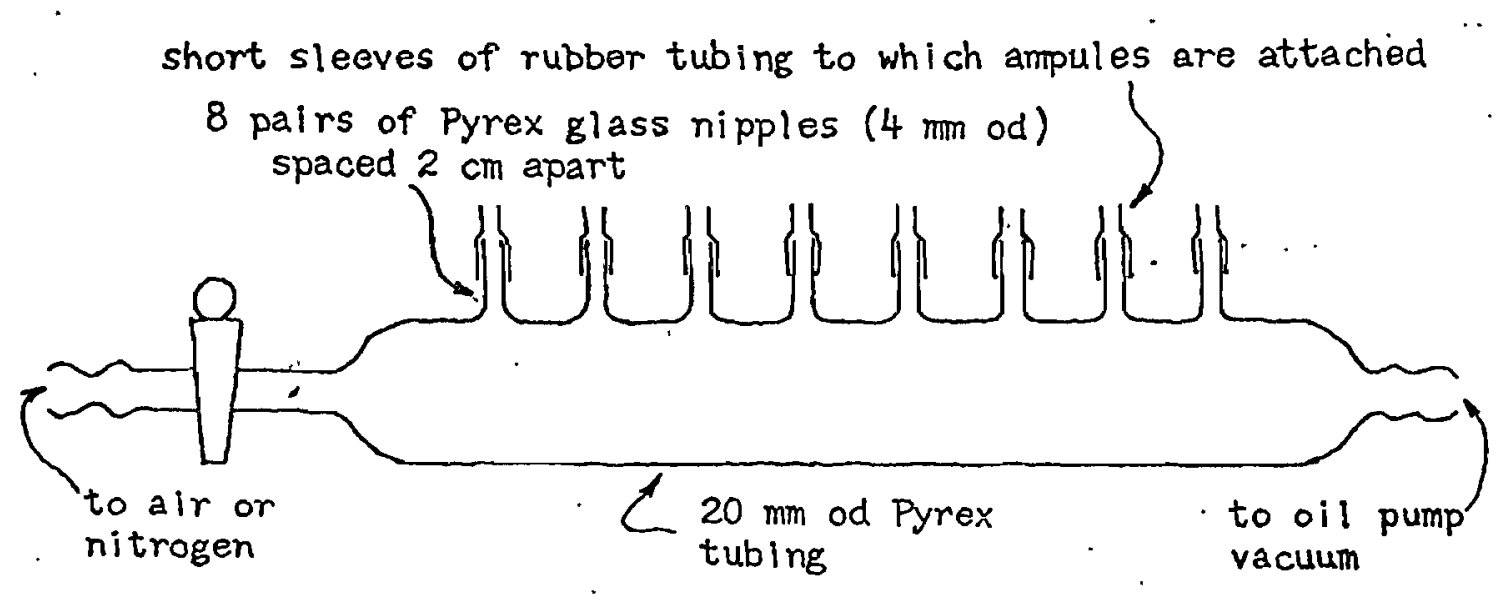

Figure 13. Apparatus for evacuating ampules. 
Experiments designed to test reproducability in concentrations of samples prepared by the method given above were carried out using a standard $5 \times 10^{-5} \mathrm{M}$ solution of trans, trans-1,6-diphenylhexa-1,6. diphenylhoxa-1,5-diene. It was found that although a decrease of from $0.5 \mathrm{mg}$ to $4 \mathrm{mg}$ in voight occured upon evacuation and sealing of an . ampule, no alteration of the sample's concentration could be obsorved, basod upon a comparison of the samplo's absorbance at $256 \mathrm{~nm}$ with that of a portion of the original standard solution. The ampules were then weight al by wrapping strips of sheet lead around the bases of their necks and were lowered into the bath by means of a basket comprised of two square lattices held in parallel horizontal planes spaced $2.5 \mathrm{~cm}$ apart. Each lattice was constructed from 1617 -m id brass rings which iad been silver-soldered together. Approximately $0.5 \mathrm{~cm}$ beneath the lower lattice were attached four horizontally-positioned brass rods, each located directly below the line of centers of a row of brass rings. These rods prevented any ampules from falling through the basket. Thermal stability of trans,trans-1,6-diphenylhexa-1,5-dieno. In this section are presented the results of several experiments whose purposes were to determine the extent of decomposition during a kinetics run of trans, trans-diene formed by the themal rearrangement of dl $-3,4-$ diphenylhexa-1,5-diene, and provide some insight into measures which might be taken to inhibit, partially or entirely, the decomposition. Decreases in sample abiorbances at $256 \mathrm{~nm}$ were detemined from nearly $\because$ always erratic plots of absorbance versus time, and are reported as percentages calculated from the expression $\left[\frac{A_{\text {init }}-A_{\text {final }}^{t}}{A_{\text {init }}}\right] 100$, where 
$A_{\text {init }}$ is tho absorbance of an unheated control sample (either experimentally letermined or calculated from the concentration of the original standzed solution) and $A_{\text {final }}^{t}$ is the absorbance of a sample heated for tine $t$.

(Run 1 at $90^{\circ}$ ) Two sets of five Pyrex glass ampules were filled with hoptane solutions of trans,trans-1,6miphenylhexa-1,5-diene, one havine a concentration of $4.27 \times 10^{-3} \mathrm{M}$ and the other $4.27 \times 10^{-5} \mathrm{M}$. After filling the ampules were chilled at $-78^{\circ}$ for at least $5 \mathrm{~min}$, evacuated to ca. 0.6 by oil pump for $4-10 \mathrm{~min}$ and flame sealed. One aipule was ramdomly selected from each set and was set aside as a control. The romaining eight ampules wexe lowered simultaneously into a constant tomperature bath maintained at $90.02 \pm 0.01^{\circ}$. Pairs of ampules were subsequently withdrawn from the bath at 12-hr intervals and quenched. The contents were then analyzed by UV either directly or after one-hundred fold dilution, depending upon the initial trans, trans concentration. Decreases in absorbances of $2.5 \%$ and $6.6 \%$ were observed for the concentrated trans, trans solutions (after dilution) after periods of heating of 12 and $47 \mathrm{hr}$ respectively. In corresponding. periors of time the absorbances of the dilute trans, trans solutions had declined $1.1 \%$ and $4.9 \%$ respectively.

(Run 2 at $90^{\circ}$ ) Eight ampules were rinsed twice with hoptane, (as described in the section on kinetics) heated in an oven at $140^{\circ}$ for $0.5 \mathrm{hr}$ and further dried by passage of a stream of tank nitrogen while being heated with a hot-air gun. Into the dried ampules were transferred 3-ml aliquots of a $4.92 \times 10^{-5} \mathrm{M}$ solution of trans,trans-diene in heptane. The ampules were chtJled to $-78^{\circ}$, evacuated and flame 
sealed, as in run 1, at $90^{\circ}$. One ampule was reserved as a control. The remaining seven were heated at $90.02 \pm 0.01^{\circ}$. Six of these were withdrawn from the bath at regular intervals over a 12ahr period and quenched. The seventh was heated for a total of $24 \mathrm{hr}$. The contents were analyzed by UV. A plot of absorbance versus time was prepared, which indicated decreases in absorbance of $1.4 \%$ and $4.6 \%$, respectively, over periods of 12 and $24 \mathrm{hr}$.

(Run 3 at $90^{\circ}$ ) Eighteen ampules were employed. One set of eight was rinsed with heptane as in run 2 at $90^{\circ}$. Eight others were filled with concentrated aqueous ammonia (to neutralize any acidic sites on the glass surface), heated in a steam bath $2 \mathrm{hr}$, rinsed with three portions of deionized wator, heated at $160^{\circ}$ in an oven and firally evacuated to full oil spump vacuum while still hot to remove residual moisture. One of the two remaining ampules was evacuated to full oil-pump vacuum and heated in a gas flame until a yellow sodium flare-off was observed. After being allowed to cool to room temperature while evacuated the ampule wis filled with tank nitrogen. The eighteenth ampule was not treated in any special manner. All 18 ampules were filled with the trans, trans solution employed in wun 2 at $90^{\circ}$, chilled to $-78^{\circ}$, evacuated and flame sealed. A control samplo from each set of eight ampules was set aside; the remeining 16 were heated to $90.02 \pm 0.03^{\circ}$. At 2-br intervals over a $12-\mathrm{hr}$ period one ampule from each set of eight was withciram from the constant temperature bath and quenched. The remaining four ampules wero heatedian additional $12 \mathrm{hr}$. Absorbanco readings wero determined at $256 \mathrm{~nm}$ for the 18 samples and plotted against time, Over 12 hours thosè samples which had been placed in 
heptare-rinsed ampules showed the least relative decline in absorbance (1.0\% compared to $2.6 \%$ for the ammonia-rinsed ampules). However the contents of the flame-heatod ampules displayed the least decrease in absorbarce $(1.4 \%)$ of any of the 24 hour samples. A $2.0 \%$ reduction in the atsorbance of the 24-hour sample contained within a heptane-rinsed ampule vas noted.

(Run 4 at $90^{\circ}$ ) Eight sets of three ampules each vere prepared. The first set was rinsed with heptane (2 portions). The next two sets were heated in a steam-bath $0.5 \mathrm{hr}$ with respectively, $12 \mathrm{M}$ hydrochloric acid and hot $20 \%$ aqueous sodium hydroxide. Tho acid-treated ampules were then rinsed with deionized water ( 3 portions). After being dried at $170^{\circ}$ in an inverted position and evacuated while hot to full oilpump vacuum, the three sets of ampules were filled with tank nitrogen, followed by $4.92 \times 10^{-5} \mathrm{M}$ trans, trans solution. Another set was evacuetod to full oil-pump vacuum and heated by a gas flame until a yellow sodiun flaremoff was observed. When these had cooled to room temperature they were filled first with tank nitrogen and then with $4.92 \times 10^{-5} \mathrm{~m}$ trans, trans solution. The remaining four sets, which had nct been trieated in any special way, wero filled with trans, trans solutions containing about 19 (by woight of trans,trans) of either din-butylamonium picrate, hydroquinone, acetic acid, or di-n-butylamine. (These solutions were prepared by admixing $10 \mu 1$ aliquots of saturated solutions, in heptane, of the picrate of hydroquinone, or dilute (ca $10^{-2} \mathrm{M}$ ) solutions, in heptane; either of glacial acetio acid or di-n-kutylamine, with $25-\mathrm{ml}$ portions of a $4.82 \times 10^{-5} \mathrm{M}$ trans, trans 
solution). All eight sets of ampules were chilled, evacuated and sealed as before. Two samples from each set were heated at $90.02 \pm 0.01^{\circ}$ for 6.5 and $12 \mathrm{hr}$ respectively. UV absorbance data were then collected as before. The most nearly linear decline in absorption vith time was obserred for those samples enclosed in hoptane-rinsed ampules $(0.4 \%$ at $6.5 \mathrm{hr}$, and $0.7 \%$ at $12 \mathrm{hr})$. Samples containing di-n-butylamine displayed a slight increase $(0.1 \%)$ in absorbance up to $6.5 \mathrm{hr}$ followed by a precipitous decrease (2.9\%) thereafter. Solutions taken from the sodiur: hydroxide-washed ampules also showed an increase in absorbance over $6.5 \mathrm{hr}$. amounting to $0.2 \%$. Unlike the previous example, however, the absorbance declined gradually over the next $5.5 \mathrm{hr}$ to give a net decrease of only $0.06 \%$. A nearly exponential increase in absorbance with time was observed for those samples placed in acid-washed ampules, with the final value, taken at $12 \mathrm{hr}$, exceeding the initial by $3.1 \%$. The remaining four sets of samples (prepared using flame-heated ampules, or trans, trans solutions containing either hydroquinone, di-n-butylammonium picrate, or acetic acid) displayed moderate (ca $1 \%$ in $12 \mathrm{hr}$ ), nearly linear, docreasos in absorbance with time.

(ikun 5 at $90^{\circ}$ ) This run was performed in order to determine if the anomalous behavior of trans, trans-diene heated in sodium hydroxiderinsed ampules could be reproduced.

Two sets of ampules were prepared. One set, containing three samples, was made up using heptane-rinsed ampules. (data aquired from this set served as reference points to which those obtained from the second set could be compared.) The second set consisted of five ampules which had been heated in a steam bath $1.5 \mathrm{hr}$ with $6 \mathrm{M}$ aqueous sodium 
hydroxide, drained and dried, etc., as described in the preceeding section. One sample from each set was reserved as a control. The remaining six were heated at $90.02 \pm 0.01^{\circ}$. Ampules were withdrawn from the bath at regular intervals over a 12-hr period, their contents examined by $\mathrm{W}$ and plots of absorbance versus time prepared. As in the previous run the absorbance of those samples heated within sodium hydroxidemashed ampules rose to a maximum (representing an increase of $1.2 \%$ ) over a period of $6 \mathrm{hr}$. An additional absorption minimum was also observed after 3 hr of heating.

(Run 6 at $90^{\circ}$ ) This run was indentical to run 5 , except that the base-washed ampules were given.two preliminary heptane rinses and were heated with base for $45 \mathrm{~min}$. Again the absorption of samples contained in the base-washed ampules rose to a maximum which, however, appeared after $3 \mathrm{hr}$ of heating rather than six. The increase amounted to $2.5 \%$. (Run 7 at $90^{\circ}$ ) Nine ampules were heated $1 \mathrm{hr}$ in a steam bath with $6 \mathrm{M}$ sodium hydroxide, After boing rinsed with three portions of deionized water the ampules were dried, etc., as described in run 4. The samples were placed in a bath at $90.02 \pm 0.01^{\circ}$ and were withdrawn at regular intörvals over a $12 \mathrm{mr}$ period. A plot of sample absorbance as a function of time vas constructed. However as the distribution of experimental points was very erratic no. useful information could be derived from it.

(Run 1 at $100^{\circ}$ ) Fourteen ampules were rinsed twice with heptane, and dried as usual. Four of these, were filled with $4.67 \times 10^{-5} \mathrm{M}$ trans, trans solution. The remaining 10 ampules were divided into two sets of five each. One set filled with $4: 67 \times 10^{-5} \mathrm{M}$ trans, trans solution 
which was ca $5 \times 10^{-8} \mathrm{M}$ in diphenylamine and the othor set with trans, trans solution containing about $5 \times 10^{-8} \mathrm{M}$-phenyl- $\beta$ waphthylamine. All three sets of ampules were sealed in the usual manner. UV data were accumulated and analyzed by the conventional proceedure.

Absorption maxima were noted at about 4.5 and $6 \mathrm{hr}$ for those samples to which diphenylamine and Nophenyl- $\beta$-naphthylamine, respectively, had been added. Decreases in absorbance of $0.9 \%, 1.5 \%$ and $2.2 \%$ were observed for those samples containing the naphthylamine, diphenylamine, and no inhibitor, respectively.

(Run 2 at $100^{\circ}$ ) Duplicate determinations of the variation of trans, trans absorbance with time were made using two sets of five ampules which had been rinsed only with heptane. The samples were heated for a maximum of six hours. Absorbances for the two sets of samples decreased $1.0 \%$ and $1.5 \%$ during this period. The variation of absorbance with time was found to be nearly linear for one set of samples but quite erratic for the other.

(Run 1 at $110^{\circ}$ ) Three sets of five samples each were prepared from ampules which had been rinsed only with heptane. Over a 3.5-hr period the average decrease in absorbances was $1.1 \%$.

Thermal rearrangement of $d 1-3,4$-diphenylhexa-1,5-djene at $110^{\circ}$. d]-3,4-Diphenylhexa-1,5mione $\left(3.9 \mathrm{mg}\right.$, mp $35.1-35.7^{\circ}$ in p-heptane (2 ml) was heated at $110 \pm 1^{\circ}$ for 12 hours in an evacuated Pyrex ampule. Gas-chromatographic analysis indicated the presenee of only one component, having a retention time corresponding to that of trans,trans1,6-diphenylhexa-1,5-diene. Any additional components present in amounts greater than $0.1 \%$ would have been detected undep the analysis 
conditions employed. This rearrangement is unusual in that such a clean product was obtained. The crude product of a similar isomerization carried out by Lutz (14) at $80^{\circ}$ contained $0.9 \%$ of a second component believed to be an impurity in the starting material. Apparently the dI-wiene used in the present experiment was of extraordinarily high purity.

Themal rearrangenent of meso-3,4-diphenylhexa-1,5-diene at $160^{\circ}$. A Pyrex glass ampule was filled with approximately $2 \mathrm{ml}$ of an $\underline{n}$-heptane solution of meso-3,4-diphenylhexa-1,5-diene (mp 86.3-86.5 ) having a concentration of $7.5 \mathrm{mg} / \mathrm{ml}$ : The ampule was chilled to $-78^{\circ}$, evacuated by oil. pump to about $0.6 \mathrm{~mm}$ and fllame sealed. The ampule was heatod in an oil bath at $160 \pm 2^{\circ}$ for $8 \mathrm{hr}$ after whj.ch time it was opened and the contents examined by glpc. Three components having retention times corresponding to trans, trans- and cis,trans-1,6-diphenylhexa-1,5-diene, and trans-1,4-diphenylhexa-1,5-diene, were detected. These represented, respectively, $42.8 \%, 56.2 \%$, and $1.0 \%$ of the total peak area. At $125^{\circ}$ the percentages are $36.9 \%, 62.7 \%$, and $0.2 \%$, respectively, the remain$\operatorname{der}(0.2 \%)$ being comprised of two uncharacterized components. Under the analytical conditions employed any components present to the extent of $0.1 \%$ or more would have been observed.

Thermal isomerization of meso-3,4-diphenylhexa-1,5-diene at $220^{\circ}$. meso-3,4-Diphenylhexa-1,5-diene (ca $10 \mathrm{mg}, \mathrm{mp} 85.7-86.2^{\circ}$ ) was placed in a Pyrex melting point capillary which was subsequently evacuated to full oji-pump vacuum and flame sealed. The capillary was transferred to a bath-type melting-point apperatus, hoated to $220^{\circ}$ during a period 
of about $3 \mathrm{~min}$ and held at $220 \pm 2^{\circ}$ for an additional $15 \mathrm{~min}$. Gasm chromatographic analysis of the rearranged material indicated the following product composition, based upon rotention times and relative peak areas: $50.4 \%$ cis,trans $-1,6$ diphenylhexa-1,5-djene, $47.3 \%$ trans, trans 1,6-diphenylhexa-1,5-diene and 2.2\% trans-1,4-diphenylhexa-1,5-diene. Three additional unidentified components having respective retention times of $1.3,2.8$ and $6.9 \mathrm{~min}$ and representing approximately $0.01,0.05$, and $0.2 \%$ of the crude product were also observed (Fig. 15). (That component appearing at 6.9 min was nearly obscured by cis, trans.) No other components, including cis,cis-1,6-diphenylhexa-1,5-diene, were discerned under the analysis conditions, which would have permitted the detection with ease of components present in amounts of $0.1 \%$.

Calatyzed rearrangemont of dl-3,4-diphenylhexa-1,5-diene on alunina. dl-3,4-Diphenylhexa-1.5-diene (100 mg, mp 32.5-34.20, lit. $35.1-35.4$ (14) was placed as a solid on a column of Woelm grade super I neutral alumina $(10.8 \mathrm{~g}$, in reagent heptane). The column was eluted with heptane $(500 \mathrm{ml}$, analytical. reagent grade) for two hours, during the course of which the columr aquiied a yellow-orange color. Thereafter elutions with $4: 1 \mathrm{v} / \mathrm{v}$ heptane-benzené (200. nI). benzene (80. nl), and methanol (100 ma) were carrich sut. Each of the four eluate fractions was evaporated to dryness in vacuum and the residues dried on full oil-pump vacuum. Infrared analysis of the oily heptane residue $(13 \mathrm{mg})$ indicated it consisted nearly exclusively of unreacted dl-diene. A relatively large amount of. solid matarial $\left(82 \mathrm{mg}, \mathrm{mp} 67-73^{\circ}\right)$ comprised of approximately $50 \%$ trans, trans-1,6-diphonylhexa-1,5-diene, (1it. mp 78.5-79.0 (14) and 50\% 
unreacted dl-diene, was obtained from the 4:1 heptanembenzene eluates. (This analysis was based upon IR data.) Chromatography by glpo of this residuo gave a single peak having a retention time corresponding to that of the trans, trans-diene. Under the analysis conditions any residual d]-diene would have rearranged quantitatively to trans, trans (14). Any remaining components were present to an extent of less than $0.1 \%$. The benzene eluate deposited less than $1 \mathrm{mg}$ of material. Owing to its size this residue was not examined further. From the methanol eluate was obtained $4 \mathrm{mg}$ of a yellow-crange oil possessing a pronounced odor. Examination of this residue by IR indicated the absence of olefinic linkages. No further characterization were attempted. The total recovery of material was $99 \mathrm{mg},(99 \%)$ of which $41 \%$ was trans, trans $-1,6 \mathrm{~m}$. diphenylhexa-1,5-diene.

Catalyzed rearrangement of meso-3,4-diphenylhexa-1,5-diene on

alumina. (Run 1) meso-3,4-Diphenylhexa-1,5-diene (100 mg, mp 86.2$86.8^{\circ}$, lit. $86-87^{\circ}$ (14) was placed as a solid on a column of Woelm grade super I neutral alumina $(10.2 \mathrm{~g}$ in reagent heptane).. The column was eluted first with heptane (reagent, $550 \mathrm{ml}$ ) over a 9-hr period, (the column became discolored) followed by benzene $(150 \mathrm{ml}$ ) and methanol (250 ml): Evaporation to dryness of the heptane eluate yielded a total of $68 \mathrm{mg}$ of solid, mp $85.1-86.1^{\circ}$, sintering at $75^{\circ}$. Similarly $25 \mathrm{mg}$ of solid, mp $76.6-84.0^{\circ}$, sintering at $68^{\circ}$, was obtained from the benzene eluate. Infrared examination indicated the residues consisted of approximately $75 \%$ unreacted meso-diene." The remainder presumably was a mixture of cis,trans- and tran.trans-1,6-diphenylhexa-1,5-diene since 
absorption characteristic of the transmdisubstituted double bonds present in these compounds was observed at $10.37 \mu$. No detectable residue remained after evaporation of the methanol eluate. The total recovery of material was $93 \mathrm{mg}(93 \%)$.

As the sole purpose of this study was to determine whether or not meso-diene was subject to rearrangoment on an alumina column no serious attempt was made to evaluate either the extent of reaction or the nature of the products formed. These investigations were deferred to a subsequent experinent winich is described below.

(Run 2) meso-3,4-Diphenylhexa-1,5-diene (200 mg, mp 85.7-86.2\%, sintering at $80^{\circ}$ ) dissolved in $5 \mathrm{ml}$ of heptane was transferred to a column of Woelm grade super I neutral alumina (18.9 $\mathrm{g}$ in reagent hep$\tan \theta$ ). The column was then eluted with $100 \mathrm{ml}$ of reagent heptane (to assure distribution of the diene along the column), securely stoppered, and let stand 70 hours. (This was the time required for the column to develop an orange discoloration whose intensity was comparable to that observed during the dl-dienes' rearrangement.) Thereafter the column was eluted successively with reagent heptane (650- and 500-ml portions). 4:1 ( $\mathrm{rol}$ ) heptáne-benzene $(500 \mathrm{mll}), \cdot$ benzene $(200 \mathrm{ml})$ and methanol $(500 \mathrm{ml})$. The two heptane eluates yielded $73 \mathrm{mg}$ of material, mp 85.5$86.7^{\circ}$, and $21 \mathrm{mg}$ of solid, mp $82.9-84.8^{\circ}$, respectively. Neither residue was further characterized. The 4:1 heptane-benzene eluates, collected in four 125-ml fractions, were evaporated in vacuum and the residues from the last three were combined.' The first 125-m fraction liberated $78 \mathrm{mg}$ of a semisolid consisting of $30.0 \%$ cis, trans-, $3.2 \%$ trans, trans-, and $0.4 \%$ cis, cis-1,6-diphenylhexa-1,5-dienes, and $60.7 \%$ 
unreacted meso-diene, based upon glpc analysis (Fig. 16). The molar ratio of cis, trans to trans, trans isomers generated solely by rearrangement on the alumina column was 9:1, calculated from glpc data. (Correction for cis, trans- and trans,trans-dione formed by rearrangement of residual meso while on the glpe column vere applied.) Ton milligrams of material, mp $63-73^{\circ}$ (turbid), was recovered from the remaining $375 \mathrm{ml}$ of heptane-bensene eluate and was not further characterized. The benzero and methanol eluates yielded respectively, $3 \mathrm{mg}$ of a semisolia, which was not further characterized and $37 \mathrm{mg}$ of a turbid, yellow oil. possessing a pronounced odor. The latter was found by IR to be similar in corposition to an oily substance isolated from the methanol eluate from a previously attempted rearrangement of di-diens. The extent of rearrangement was calculated to be about 15\%: $220 \mathrm{mg}$ of material. were recovered, representing an increase of $10 \%$ over the weight of material originally placed on the column. 


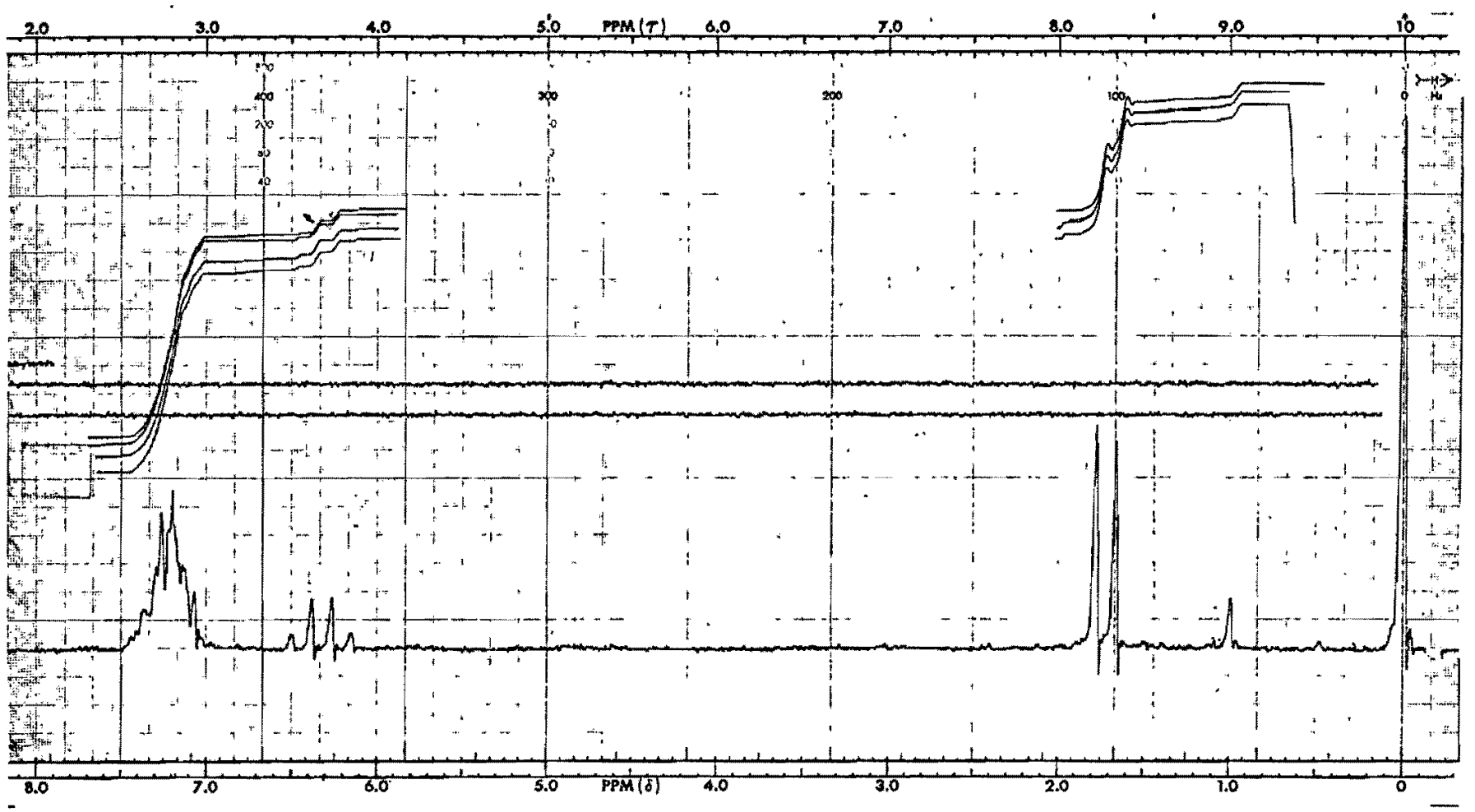

Figure 14. MR spectrum of crude product from attempted preparation of meso-3,4-diphenylhexa-1,5-diene during which base-catalyzed rearrangement occurod. 


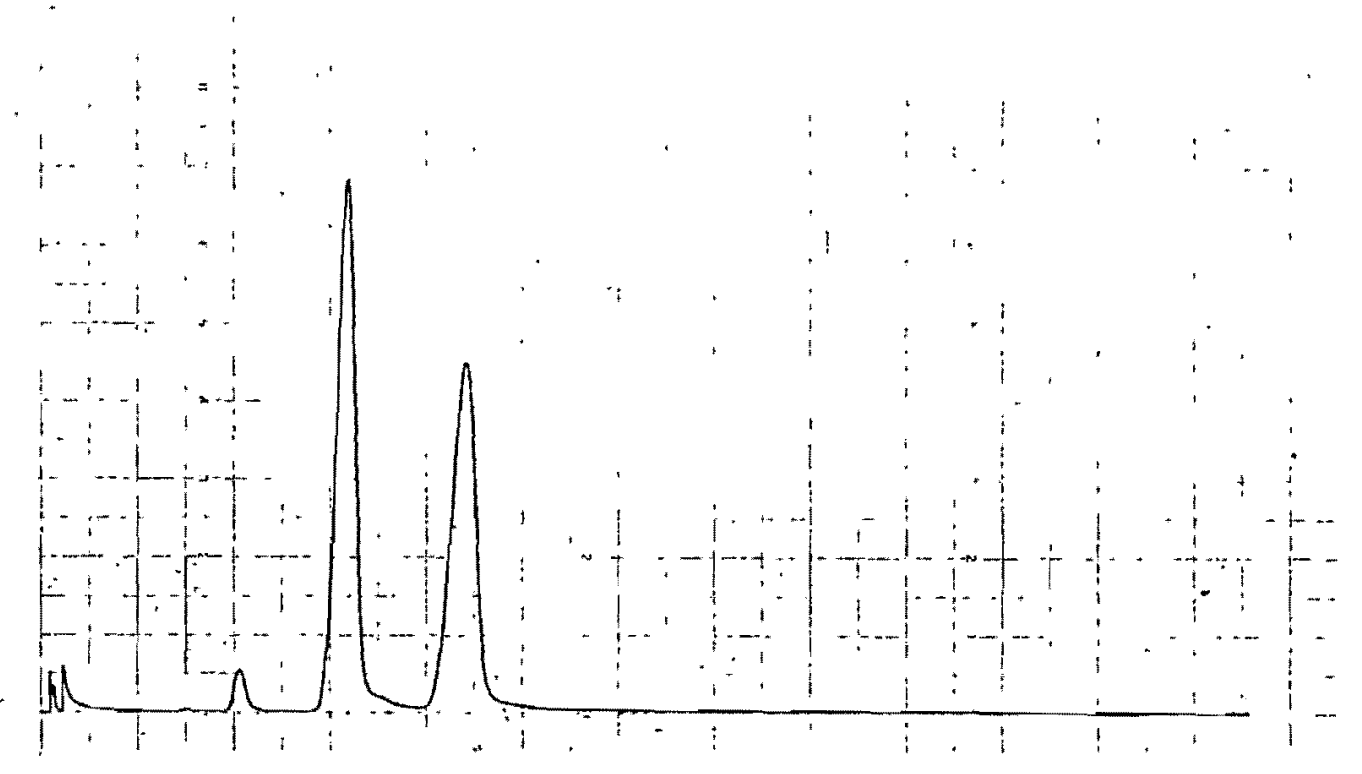

Ficure 15. Gas chromatogram of meso-3,4-diphenylhexa-1,5-diene rearranged neat at $220^{\circ}$ for 15 min.

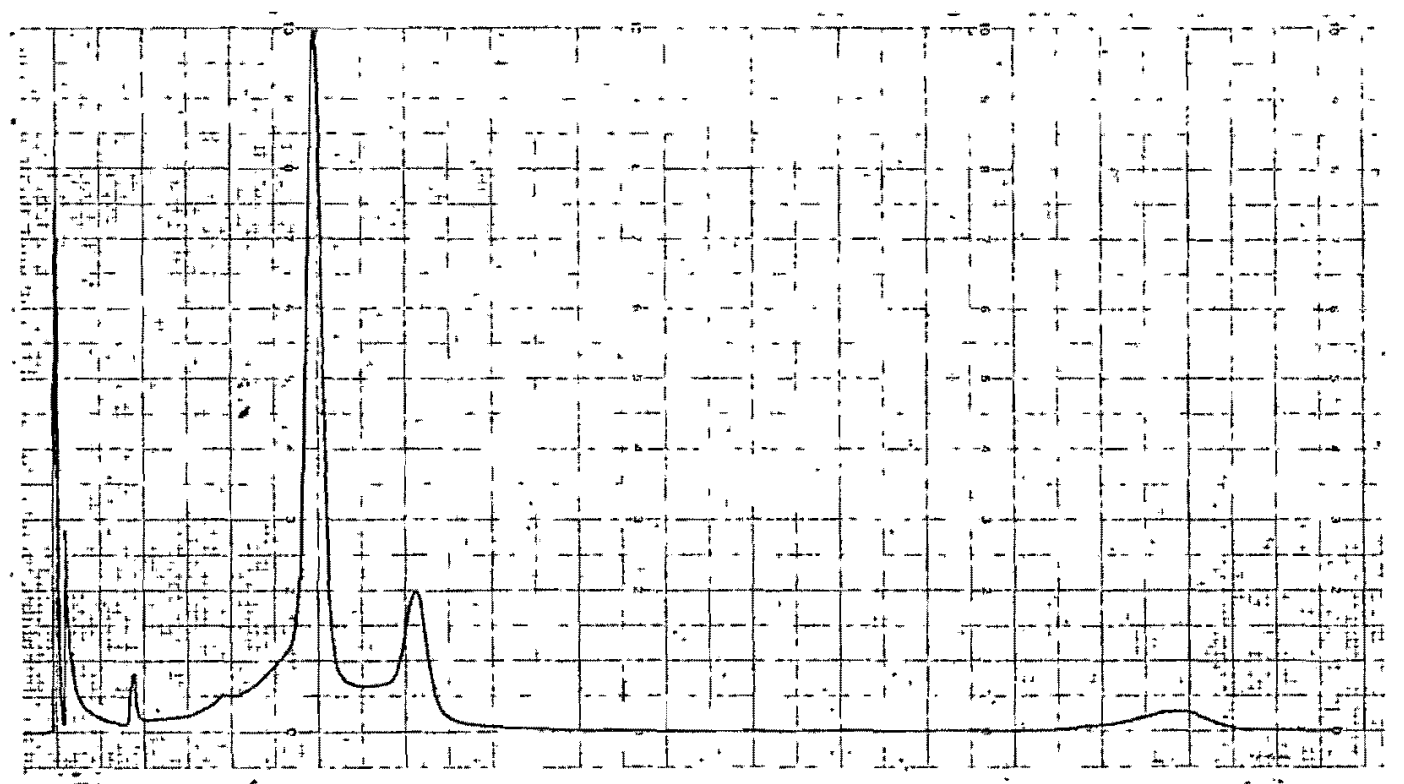

Iipure 16. Gas chromatogram of the 4:1 v/v heptane-benzene eluate residue from rearrangement of meso-3,4-diphanylhexa-1,5-diene on al umina. 


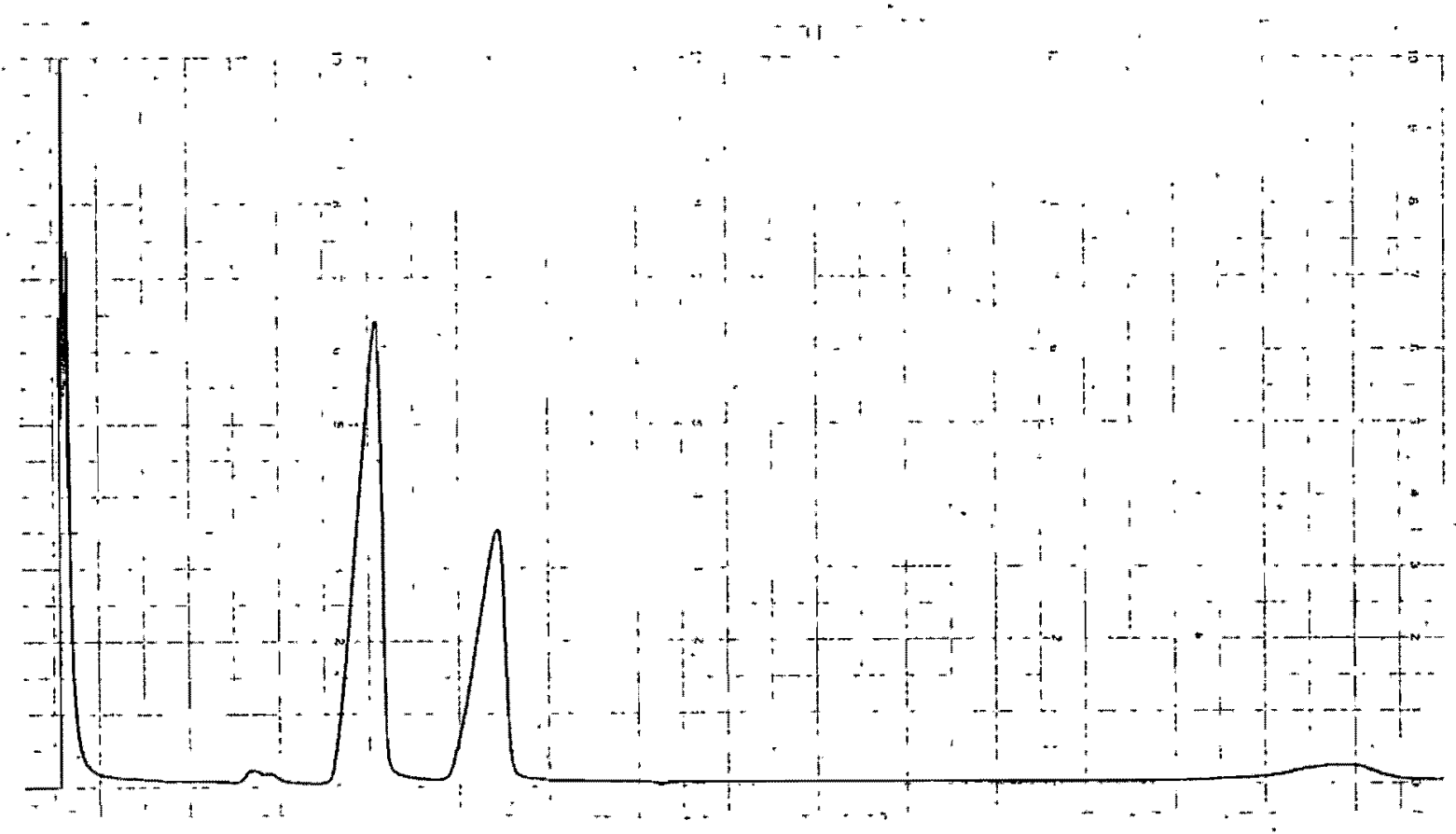

Figure 17. Gas chromatogram of heated $\left(220^{\circ}, 15 \mathrm{~min}\right) 4: 1 \mathrm{v} / \mathrm{v}$ heptane-benzene eluate residue from rearrangenent of meso-3,4diphenylhexa-1,5-diene on alumina. 
REFERENCES

$1 \nabla$. Voorhees and G, S. Skinner, J. An, Chem, Soc, 47, 1124 (1925).

2 A. C. Cope and S. M. MCEJvain, J. Ars. Chem. Soc.s 54, 4311 (1932).

3 A. G. Cope and I. M. Hancock, J. Am. Chem. Soc., 60, 2644 (1938); ibid, 60, 2901 (1938); ibid, 60, 2903 (1938).

4 A. C. Cope and E. H. Eardy, J.Am, Chem, Soc., 62, 441 (1940).

5 A. C. Cope, K. E. Hoyle, and D. HeyJ, J. Am. Chem. Soc., 63, 1843

6 D. E. Thyte and A. C. Cope, J. Am. Chem. Soc., 65, 1999 (19433).

7 H. Levy and A. C. Cope, J. Am. Chem. Soo., 66, 1684 (1944).

8 A. C. Cope, C. M. Hofmann, and E, M. Herdy, J.Am. Chem. Soco, 63, $1852(1941)$.

9 S. J. Rhoads in "Molecular Rearrangements," P. de Mayo, Ed., Vol 1, Interscience, New York, N. Y., 1963, Chapter 11.

10 D. G. Wigfield and S. Feiner, Can. J. Chem., 48, 855 (1970).

11 C. Walling and M. Waiman, J. Am. Chem. Soc., 84, 2628 (1962).

12 W. von E. Doering and W. R. Rötrt, Tetrahedron, 18, 67 (1962).

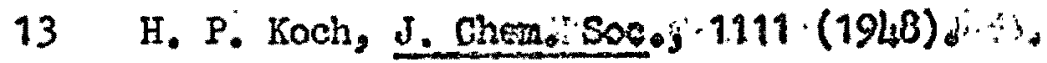

14 R. P. Lutz, S, Bermal, R. J. Boggio, R. O. Harris, and $H_{*} W_{0}$ McNicholas, J. Am. Chem. Soc., 93, 3985 (1971).

15 M. M. Baizer and J. D. Anderson, J. Electrochem. Soc., 111, 223 (1964).

16 E. J. Corey and S. Terashima, Tetrahedron Lett.s: 111 (1972).

17 W. von E. Doering, F. G. Toscano, and G. H. Bèsley, Tetrahedron, 27, 5299 (1971): ; :

18 W. von E. Doering, M. Franklk-Neumann, D. Hasselmann, and R. L. Kaye, J. Am.Chem. Soc.; gL, 3833 (1972)。 
19 I. Amcur and G. G. Hanmes, "Chemical Kinetics: Principles and Selected Topics," McGrar-Hij]I, New York, N. Y., 1966, Chapter 2。

20 Q. R. Aldridge and G. W. Murphy, J. Am. Chem. Soc., 73, 1158 (1951).

21 H. von E. Doering, B. M. Ferrier, E. To Fossel, J. H. Hartenstein, $M_{*}$ Jones Jr., G. Klumpp, $R_{6} M_{*}$ Rubin, and $H_{*}$ Saunders; Tetrahedron, 23, 3943 (1967).

22 P. S. Wharton and R. A. Kretchmer, J. Org. Chem., 33, 4258 (1968).

23 A. Viola and J. H. Mackizlan, J. Am. Ghem. Soc., 92, 2404 (1970).

24 Wo von Eoexing, Zh. Vsesoyuz. Khim, Obshchestra im $D_{0} I_{0}$ Kendeleeva, 7,308 (1962).

25 J. C. Trebellas, J. R. Olechowski, and H. B. Jonassen, J. Organometal. Chess., 6, 412 (1966).

26 P. Heimbach and W. Brenner, Angew. Chem. Intern. Ed., 6, 800 (1967).

27 H. F. Stewart and R. P. Seibert, J. Org. Chem., 33, 4560 (1968).

28 M. P. Oommen and A. I. Vogel, J. Chem. Soc., 2148 (1930).

29 A. G. Brook, H. L. Cohen, and G. F. Wright, J. Org. Chem., 18, 447 (1953).

30 B. Loev and M. M. Goodman, Ghem, Ind. (London), 2026 (1967).

31 H. H. Jaffe and M. Orchin, "Theory and Applications of Untraviolet Spectoscopy," Wiley, New York, N. Y., 1962, Chapter 20. 
APPENDIX A

RAW KINETICS DATA

TABLE III

FIRST RUN AT $90^{\circ}$

Absorbance

0.382

0.641

0.850

1.030

1.153

1.264

1.387

1.491

1.544

1.574

1.615

1.654

$: 1.677$

$$
1.636
$$

Time, hr

1.01

2.00

2.98

3.99

5.00

6.00

7.16

8.99

10.02

11.01

12.01

13.00

13.99

48.01

dl-Diene conc $=4.98 \times 10^{-5} \mathrm{M}$

Temperature, corrected $=89.89^{\circ}+0.14^{\circ}$

$\therefore=90.03^{\circ}$

Rate constant $=5.44 \times 10^{-5}: \mathrm{sec}^{-1}(4$ half-lives $)$

$$
=5.54 \times 10^{-5} \mathrm{sec}^{-1} \text { (3 half-lives) }
$$




$$
\begin{aligned}
& i \\
& \text { in } \\
& \text { TABLE IV } \\
& \text { SECOND RUN AT } 90^{\circ} \\
& \text { Absorbance } \\
& 0.441 \\
& +\quad .0 .746 \\
& 0.986 \\
& 1.173 \\
& 1.334 \\
& 1.416 \\
& 1.475 \\
& 1.525 \\
& 1.563 \\
& 1.600 \\
& 1.625 \\
& 1.640 \\
& =90.03^{\circ} \\
& =.5 .46 \times 10^{-5} \mathrm{sec}^{-1} \text { (3 half-lives) }
\end{aligned}
$$


TABLE V

THIRD RUN AT $90^{\circ}$

Absorbance

0.348

0.604

0.818

0.989

1.127

1.244

1.335

1.411

1.476

1.519

1.569

1.601

1.631

1.641
Time, hr

1.00

2.00

3.00

4.00

5.00

6.04

7.00

8.00

9.00

10.00

11.00

12.17

13.00

14.00

dl-Diene conc $=4.93 \times 10^{-5} \mathrm{M}$

Temperatüre, corrected $=89.76^{\circ}+0.14^{\circ}$

$$
=89.90^{\circ}
$$

Rate constant $=5.34 \times 10^{-5} \mathrm{sec}^{-1}$ (4 half-lives)

$$
=5.51 \times 10^{-5} \sec ^{-1} \text { (3 half-lives) }
$$


TABLE VI

FIRST RUN AT $100^{\circ}$

Absorbance

0.422

0.692

0.928

1.095

1.232

1.346

1.426

1.490

1.547

1.569

1.614

1.624

1.658
Time, hr

0.536

1.000

1.528

2.000

2.500

3.000

3.500

4.000

4.526

5.000

5.508

6.000

6.500

dl-Diene conc $=4.84 \times 10^{-5} \mathrm{M}$

Tomperature, corrected $=99.71^{\circ}+1.6 \times 10^{-4}\left(17^{\circ}\right)\left(99.71^{\circ}-31.7^{\circ}\right)$

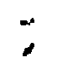

$$
+0.11^{\circ}
$$

$$
=100.01^{\circ}
$$

Rate constants $=1.31 \times 10^{-4} \sec ^{-1}(4.5$ half-lives $)$

$$
=1.36 \times 10^{-4} \sec ^{-1} \text { (3 half-lives) }
$$


TABLE VII

SECOND RUN AT $100^{\circ}$

\begin{tabular}{cc} 
Absorbance & Time, $\mathrm{hr}$ \\
\hline 0.434 & 0.550 \\
0.690 & 1.000 \\
0.919 & 1.500 \\
1.094 & 2.000 \\
1.231 & 2.500 \\
1.345 & 3.000 \\
1.427 & 3.500 \\
1.497 & 4.000 \\
1.554 & 4.550 \\
1.589 & 5.000 \\
1.612 & 5.500 \\
1.626 & 6.000 \\
1.657 & 6.500 \\
1.669 & 6.933
\end{tabular}

d1-Diene conc $=4.84 \times 10^{-5} \mathrm{M}$

Temperatjure, corrected $=99.73^{\circ}+1.6 \times 10^{-4}\left(17^{\circ}\right)\left(99.73^{\circ}-34.5^{\circ}\right)$

$$
\begin{aligned}
& +0.11^{\circ} \\
= & 100.02^{\circ}
\end{aligned}
$$

Rate constant $=1.32 \times 10^{-4} \sec ^{-1}(5$ half-lives $)$

$$
=1.38 \times 10^{-4} \mathrm{sec}^{-1} \text { (3 half-lives) }
$$

Samples contained approximately $5 \times 10^{-8} \mathrm{M} N$-phenyl- $\beta$-naphthylamine. 
TABLE VIII

THIRD RUN AT $100^{\circ}$

Absorbance

0.419

0.708

0.933

1.109

1.249

1.350

1.440

1.503

1.552

1.592

1.623

1.667

1.675
Time, $h r$

0.500

1.000

1.502

2.000

2.500

3.000

3.500

4.000

4.500

5.000

5.508

. 6.500

6.500

dl-Diene conc $=4.86 \times 10^{-5} \mathrm{M}$

Temperature, corrected $=99.74^{\circ}+1.6 \times 10^{-4}\left(17^{\circ}\right)\left(99.74-38.0^{\circ}\right)$

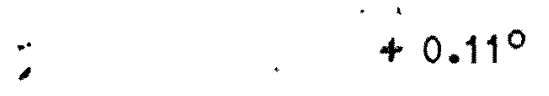

$$
=100.02^{\circ}
$$

Rate constant $=1.36 \times 10^{-4} \sec ^{-1}(4.5$ Half-lived $)$

$$
=1.36 \times 10^{-4} \mathrm{sec}^{-1}(3 \text { half-lives })
$$


TABLE IX

FOURTH RUN AT $100^{\circ}$

Absorbance

0.420

0.707

0.930

1.108

1.257

1.360

1.437

1.502

1.553

1.605

1.616

1.649

1.672

1.688
Time, $h r$

0.500

1.000

1.500

2.000

2.501

3.065

3.500

4.000

4.500

5.000

5.500

6.000

6.502

7.049

d]-Diene conc $=4.86 \times 10^{-5 \mathrm{M}}$

Temperațure, corrected $=99.74^{\circ}+1.6 \times 10^{-4}\left(17^{\circ}\right)\left(99.74-38.0^{\circ}\right)$

$$
+0.11^{\circ}
$$$$
=100.02^{\circ}
$$

Rate constants $=1.37 \times 10^{-4} \sec ^{-1}$ ( 5 half-Iives $)$

$$
=1.36 \times 10^{-4} \mathrm{sec}^{-1} \text { ( } 3 \text { half-lives) }
$$

Samples contained $5 \times 10^{-7} \cdot \mathrm{M}$ N-phenyl- $\beta$-raphthylamine. 
TABLE X

FIFTH RUN AT $100^{\circ}$

\section{Absorbance}

0.432

0.716

0.946

1.116

1.310

1.362

1.437

1.509

1.555

1.597

1.625

1.644

1.676
Time, $\mathrm{hr}$

0.500

1.000

1.500

2.000

2.500

3.000

3.500

4.002

4.500

5.000

5.500

6.000

6.500

dl-Diene conc $=4.86 \times 10^{-5} \mathrm{M}$

Temperature, corrected $=99.74^{\circ}+1.6 \times 10^{-4}\left(17^{\circ}\right)\left(99.74-38.0^{\circ}\right)$

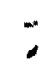

$$
\begin{array}{r}
+0.11^{\circ} \\
=100.02^{\circ}
\end{array}
$$

Rate constants $=1.36 \times 10^{-4} \mathrm{sec}^{-1}$ (4.5 half-lives)

$$
=1.38 \times 10^{-4} \mathrm{sec}^{-1} \text { (3 half-lives) }
$$


TABLE XI

FIRST RUN AT $110^{\circ}$

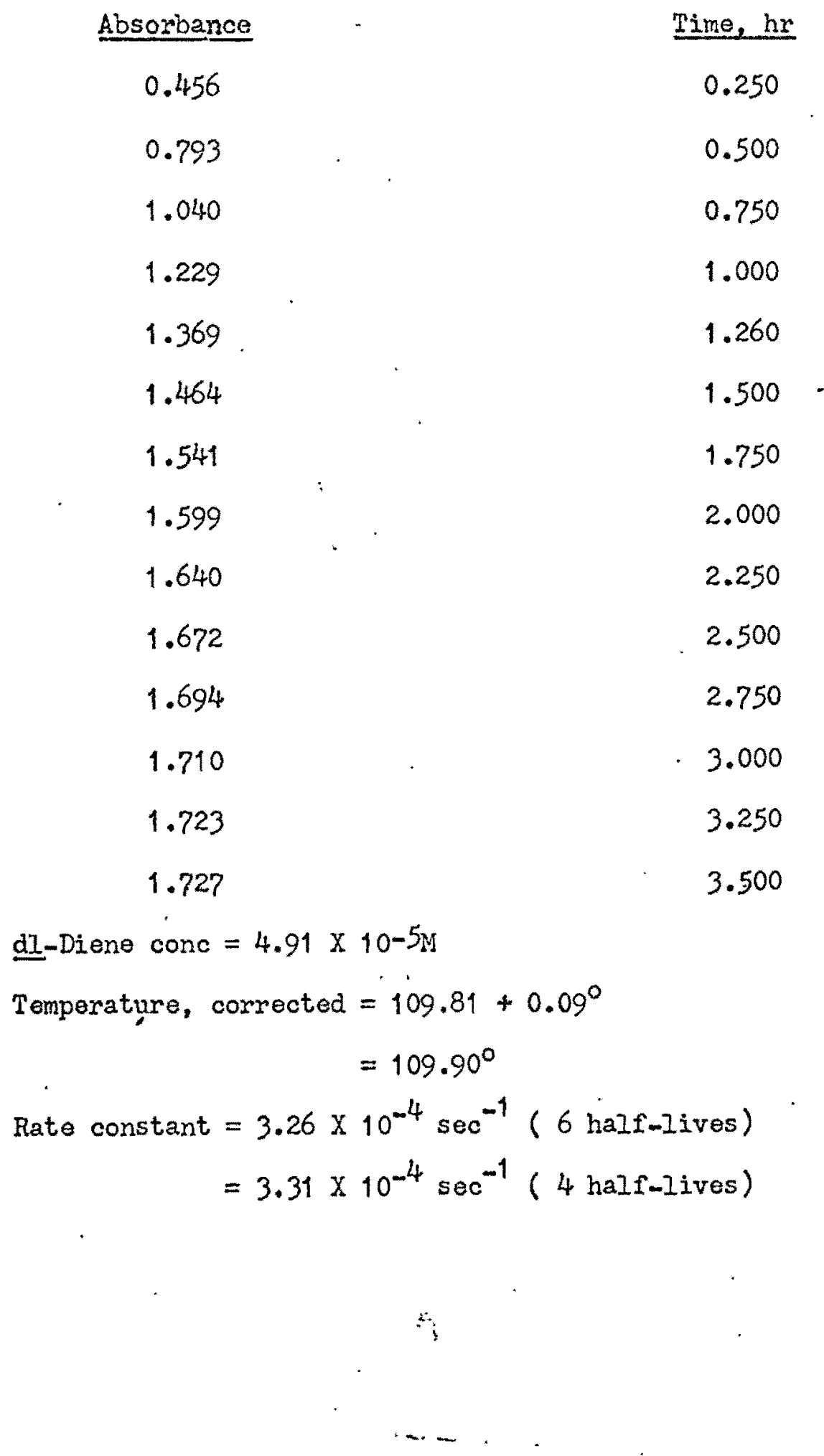


TABLE XII

SECOND RUN AT $110^{\circ}$

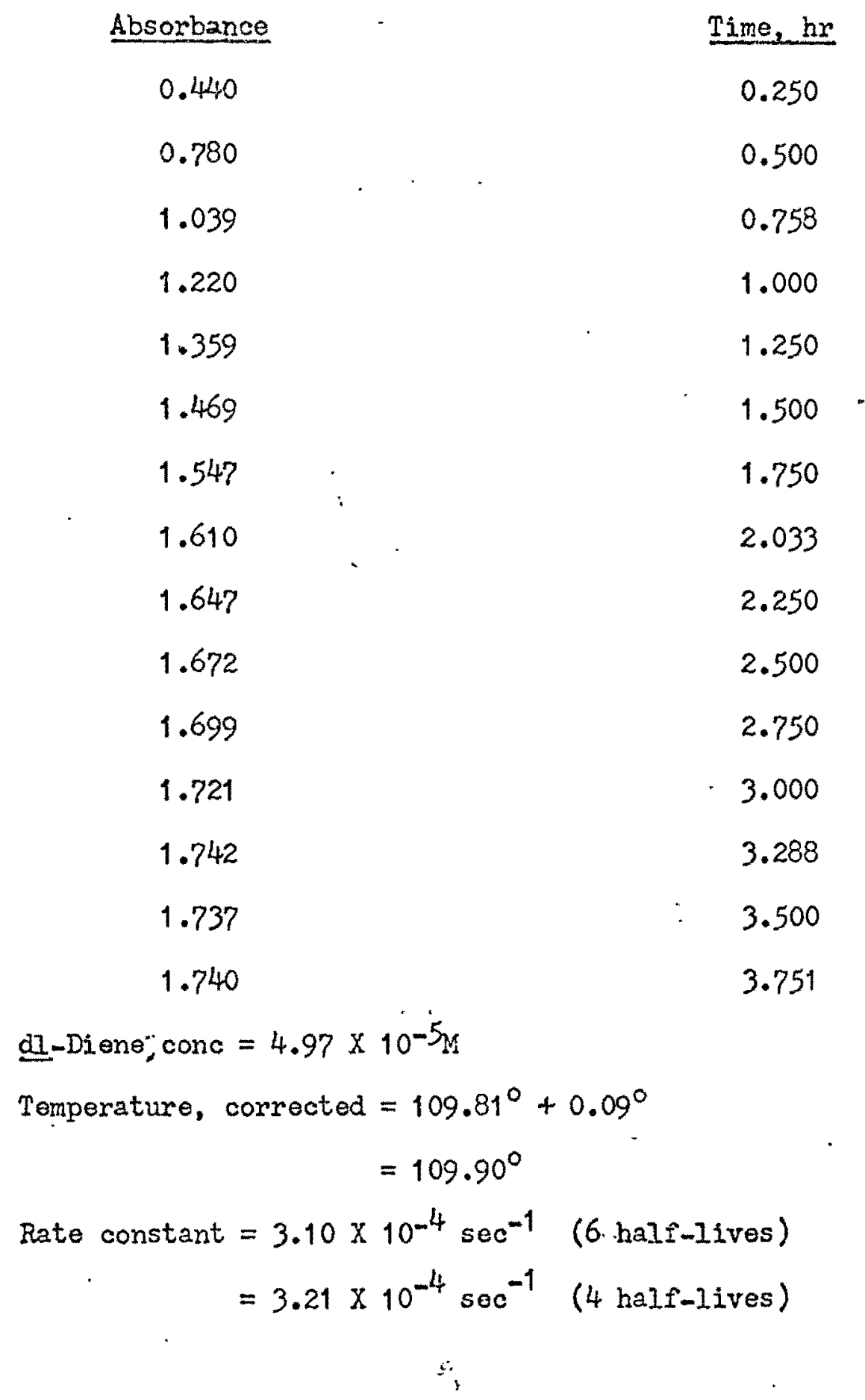


TABLE XIII

THIRD RUN AT $110^{\circ}$

Absorbance

0.453

0.790

1.035

1.227

1.359

1.470

1.556

1.595

1.638
Time, hr

0.250

0.500

0.750

1.000

1.250

1.500 .

1.750

2.000

2.250

dl-Diene conc $=4.97 \times 10^{-5} \mathrm{M}$

Temperature, corrected $=109.80^{\circ}+0.09^{\circ}$

$$
=109.89^{\circ}
$$

Rate constant $=3.15 \times 10^{-4} \mathrm{sec}^{-1}$ (4 half-lives)

Surface-to-volume ratio increased 8-fold. 
APPENDIX B

\section{EVALUATIOINS OF KTNETICS DATA \\ AID ACTIVATION PARANETERS}

First-order rate constants were evaluated graphically via the integrated form of the first-order rate equation $-\ln \left(c / c_{0}\right)=k t(19)$, where $c / c_{0}$ is the fraction of reactant remaining after time $t$, and $k$ is the specific reaction rate constant. Values of $-\ln \left(c / c_{0}\right)$ vere determined directly by computer from sample absorbances measured in the $\mathrm{WV}$ at $256 \mathrm{~mm}$ using the expression $-\ln \left\{\mathrm{c}_{0}-\left[\left(\mathrm{A}-674.4 \mathrm{c}_{0}\right) / 35160\right]\right\}+\operatorname{In} \mathrm{c}_{0}$. The latter formulation is derived as follows. The total absorbance at any wavelength of a binary mixture is given by $A=\left(\epsilon_{1} c_{1}+\epsilon_{2} c_{2}\right)$ b whore $A$ is the total absorbance, $b$ is the pathlength of the sample, $\epsilon_{1}$ and $\epsilon_{2}$ are the molar extinction coefficients for components 1 and 2 respectively, and $c_{1}$ and $c_{2}$ are the corresponding molar concentrations (31). In the rearrangement of dl-3,4-diphenylhexa-1,5-diene the molar concentrations of product and unreacted starting material are related by the expression $c_{0}=c_{1}+c_{2}$ where $c_{1}$ and $c_{2}$ are the molarities of trans, trans-1,6diphenylhexa-1,5-diene and dz-3,4-diphenylhexa-1,5-diene at any time during reaction, and $c_{0}$ is the initial molarity of dl-diene. (This relationship is seen to be valid since a single product is formed, and then only in a 1:1 stoichiometric ratio with the starting material.) Substitution of $c_{2}=c_{0}-c_{1}$ from the last equation into the expression immediately preceeding it followed by rearrangement gives as. the concentration of trans, trans after any period of reaction the value 
$c_{1}=\frac{1}{\epsilon_{1}-\epsilon_{2}}\left[\frac{A}{b}-\epsilon_{2} c\right]$. The concentration of residual dl-diene is thus seen to be $c_{2}=c_{0}-c_{1}=c_{0}-\frac{1}{\epsilon_{1}-\epsilon_{2}}\left[\frac{A}{b}-\epsilon_{2} c_{0}\right]$. Values of $-\ln \left(c_{2} / c_{0}\right)$ may then be calculated from $-\ln \left(c^{2} / c_{0}\right)=-\ln c_{2}+\ln c_{0}=$ $-\ln \left\{c_{0}-\frac{1}{\epsilon_{1}-\epsilon_{2}}\left(\frac{A}{b}-\dot{\epsilon}_{2} c_{0}\right)\right\}+\ln c_{0}$ Experimentally determined extinction coefficients having the values $\epsilon_{2}=674.4 \mathrm{~cm}^{-1}$ molar -1 and $\epsilon_{1}=$ $3.58 \times 10^{4} \mathrm{~cm}^{-1}$ molar ${ }^{-1}$ were substituted into the preceeding equation to give the final expression $-\ln \left\{c_{0}-\frac{1}{35160}\left(A-674.4 c_{0}\right)\right\}+\ln c_{0}$.

(A value of $1.000 \mathrm{~cm}$ was assigned to the pathlength b.)

Enthalpies of activation were calculated from the temperature dependence of the rate constant under isobaric conditions. Plots of $-I n(k / T)$ versus $1 / T$, where $k$ is the rate constant at absolute temperature $T$, were constructed and their slopes evaluated by means of a least-squares computer program-(19). Activation entropies were evaluated from the equation $k=\frac{k T}{h} \cdot \exp \left(-\frac{\Delta H^{*}}{R T}\right) \exp \left(\frac{\Delta S^{*}}{R}\right)$ there $k$ is the rate constant at the absolute temperature $T$, $k$ is. Boltzmann's constant, $h$ is Planck's constant, $R$ is the gas constant, and $\Delta H^{\ddagger}$ and $\Delta S^{*}$, are the enthalpy and entropy of activation, respectively-(19). 